\title{
Kernos
}

Revue internationale et pluridisciplinaire de religion grecque antique

16 | 2003

Varia

\section{Apollon, le scorpion et le frêne à Claros}

\section{Philippe Monbrun}

URL : http://journals.openedition.org/kernos/817

DOI : $10.4000 /$ kernos. 817

ISSN : 2034-7871

\section{Éditeur}

Centre international d'étude de la religion grecque antique

\section{Édition imprimée}

Date de publication : 1 janvier 2003

Pagination : 143-170

ISSN : 0776-3824

Référence électronique

Philippe Monbrun, «Apollon, le scorpion et le frêne à Claros », Kernos [En ligne], 16 | 2003, mis en ligne le 14 avril 2011, consulté le 05 mai 2019. URL : http://journals.openedition.org/kernos/817 ; DOI :

10.4000/kernos.817 


\section{Apollon, le scorpion et le frêne à Claros $^{1}$}

Dans le traité Sur la Nature des Animaux, « la collection la plus riche d'histoires d'animaux à l'époque impériale $»^{2}$, Élien rapporte une tradition insolite à propos du sanctuaire oraculaire d'Apollon à Claros ${ }^{3}$. Il écrit au début du $\mathrm{II}^{\mathrm{e}}$ siècle après J.-C., alors que ce sanctuaire jouit encore d'une grande notoriété. Élien s'appuie sur le témoignage autorisé de Nicandre de Colophon, prêtre d'Apollon Clarien à l'époque hellénistique, mais aussi naturaliste, auteur de poèmes didactiques sur les morsures des animaux venimeux et leurs antidotes: les Theriaca et les Alexipbarmaka. Voici sa notice sur Claros:

À Claros aussi le fils de Zeus et de Léto est l'objet d'un culte particulier que lui

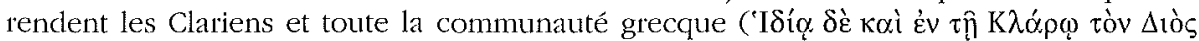

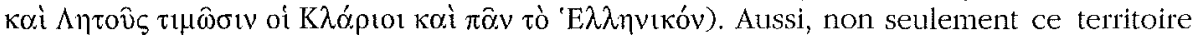
n'est-il pas fréquenté par les bêtes venimeuses, mais il leur est radicalement hostile

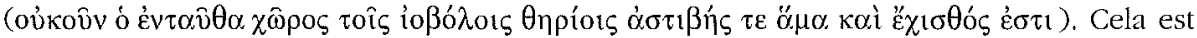

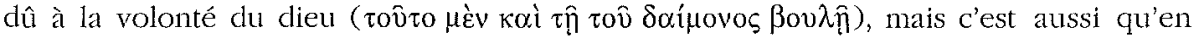
règle générale les bêtes sauvages ont terriblement peur de lui, vu qu'il sait personnel-

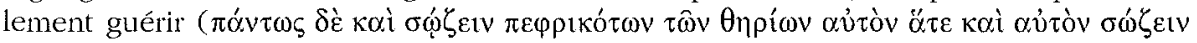

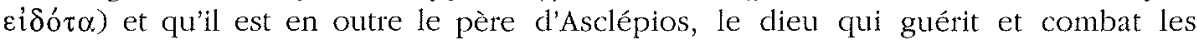

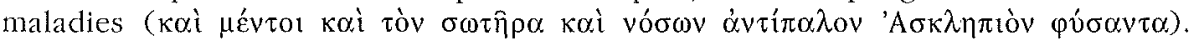
Nicandre peut témoigner aussi de la vérité de ce que je clis. Voici ce qu'il dit (å $\lambda \lambda \dot{\alpha}$

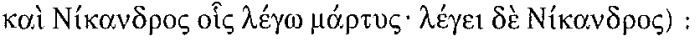

'Ni la vipère, ni les horribles araignées, ni le scorpion à la profonde morsure ne vivent dans les bois sacrés de Claros. Car Phoibos, ayant caché le profond vallon sous les frênes, a écarté les venimeux reptiles de ce sol herbeux.'

1 Je remercie chaleureusement Pierre Brulé et les membres du comité de rédaction de la revue pour la relecture qu'ils ont faite de cet article et pour leurs précieux conseils.

2 U. Dierauer, "Raison ou instinct? Le développement de la zoopsychologie antique", in B. Cassin, J.-L. Labarrière (éds), L'Animal dans lantiquité, Paris, Vrin, 1997, p. 27-28. J'utilise ici le titre latin ordinairement donné à l'ouvrage d'Élien. A. Zucker, dans sa récente traduction commentée (Paris, Les Belles Lettres, t. I, 2001 et t. II, 2002, la « Roue à Livres »), retient le titre grec Peri zôôn idiotètos qu'il rend par La Personnalité des animaux.

3 Le sanctuaire de Claros, en Ionie, était situé à $13 \mathrm{~km}$ au sud de la cité de Colophon dont il dépendait, et à $1 \mathrm{~km}$ du port de la cité, Notion ou Colophon-de-la mer. Le renom de l'oracle clarien est longtemps resté bien modeste par rapport à celui du sanctuaire voisin d'Apollon à Didymes. Il ne dépassa guère les limites de la cité de Colophon avant le début de la période hellénistique, pour ne cesser de croitre et culminer au II ${ }^{\mathrm{e}}$ siècle après J.-C. Voir, par ex., L. RoberT, Les fouilles de Claros, conférence donnée à l'Université d'Ankara en 1953 et reprise dans L. RoBERT, Opera minora selecta VI, Amsterdam, 1989, p. 538-548, ainsi que J. de LA Genière, "Claros 1992. Bilan provisoire des foulles dans le sanctuaire d'Apollon", REA 95 (1993), p. 391-392 et "Claros. Bilan provisoire de dix campagnes de fouilles", REA 100 (1998), p. 250-251. Les fouilles menées par L. Robert entre 1950 et 1961 ont permis la découverte de nombreux monuments, en particulier le temple d'Apollon avec son adyton voûté. De 1988 à 1998, le sanctuaire a été à nouveau fouillé par la mission archéologique française dirigée par $J$. de La Genière. 


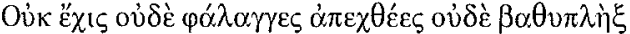

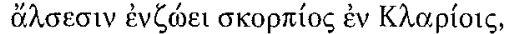

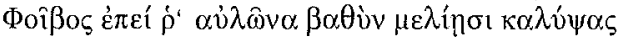

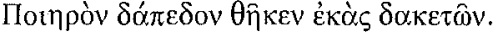

$\left(N A \mathrm{X}, 49^{4}\right)$

Sur l'absence de ces animaux, plusieur's explications ont été proposées. Charles Picard, dans son livre très critiqué sur une prétendue parenté entre les cultes d'Artémis à Éphèse et d'Apollon à Claros, a évoqué « la tradition déformée, spécialement connue à Delphes, d'une lutte d'Apollon contre le serpent chthonien $»^{5}$.

L'argumentation développée par Louis Robert me parait plus pertinente. Il a eu tout loisir de constater que le venimeux bestiaire clarien caractérisait encore récemment le site du sanctuaire : « des vipères comme ailleurs, des scorpions en quantité extraordinaire et une araignée très venimeuse que les gens appellent bügü » ${ }^{6}$. Il en vient alors à penser que « c'est justement parce que toute la région était infestée de ces bêtes, que Nicandre a relevé qu'elles ne se trouvaient pas dans le domaine même d'Apollon » et il retient deux solutions : il peut s'agir soit d'« une tradition aussi pieuse qu'erronée », soit de « l'animation perpétuelle du sanctuaire » qui chassait ces animaux ${ }^{7}$. Invoquer l'affluence des pèlerins auprès d'un oracle réputé est un argument très recevable mais cette explication rationaliste ne me paraît pas suffisante ici. La première solution avancée cerne mieux le problème. Il s'agit d'une tradition attachée au bois sacré, à l'alsos du sanctuaire oraculaire d'Apollon à Claros et qui pourrait fort bien avoir une origine apotropaïque et propitiatoire : alors que vipères, araignées et scorpions peuplent effectivement le sanctuaire et ses abords, on affirme le contraire de la réalité afin d'en mieux conjurer les dangers. On peut invoquer aussi, avec Fritz Graf, la nécessaire pureté de l'espace sacré qui se manifesterait par l'absence de ces animaux venimeux dans l'alsos de frênes couvrant le sanctuaire ${ }^{8}$.

Mais le plus important est peut-être ailleurs : la vipère ("̌̌ $\chi \varsigma)$, l'araignée appelée « phalange » $(\varphi \alpha \dot{\alpha} \lambda \alpha \gamma \xi)^{9}$ et le scorpion ( $\left.\sigma \kappa o \rho \pi i ́ \varsigma\right)$ sont à leur place dans un contexte apollinien, aux côtés du dieu à l'arc. Ce sont des prédateurs qui mordent ou piquent leurs proies et leur injectent du venin avec leurs crochets ou leur aiguillon. Ils sont munis de véritables « pointes » qu'ils plantent dans le corps de leurs victimes à la

4 J'ai utilisé la traduction de Zucker, o.c. II (n. 2), pour le premier paragraphe et celle de Robert, o.c. (n. 3), p. 528, pour les quatre vers qui forment le fragment 31 de Nicandre. J'ai repris le texte grec de la Loeb Classical Library (trad. A.F. Scholfield, Cambridge, réimpr. 1971 [1959]).

5 Ch. PICARD, Éphèse et Claros. Recherches sur les sanctuaires el les cultes de l'lonie du Nord, Paris, $1922(B E F A R, 123)$, n. 3, p. 66. Voir aussi p. 388. On peut lire le jugement sans appel que L. Robert (o.c. [n. 3], p. 546) porte sur ce travail.

6 O.c. (n. 3), p. 528. Il ajoute avec humour que « ce contraste entre l'affirmation de Nicandre et la réalité est pour les fouilleurs assez piquant». Voir aussi le témoignage de J. de La Genière dans son introduction aux Cabiers de Claros $I$, contributions qu'elle a réunies dans Ëditions Recherches sur les Civilisations, Paris, 1992, p. 13.

7 O.c. (n. 3), p. 529.

8 F. Graf, "Bois sacrés et oracles en Asie Mineure", in Les bois sacrês, Actes du Colloque International du Centre Jean Bérard et de l'EPHE (Vection), Naples, 23-25 novembre 1989, Naples, 1993 (Collection du Centre Jean Bérard, 10), p. 28.

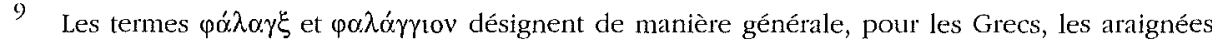
venimeuses, plus précisément la tarentule. De nombreuses occurrences dans l'Histoire des Animaux d'Aristote (notamment V, 27, 555b 10-17 et IX, 39, 622b 27-33). C'est le phalangium de Pline (HN IX, 79 et XXIX, 84-85). 
façon d'une flèche empoisonnée : l'adjectif ioßó $\lambda o \varsigma$ ('ós / $\beta \alpha \dot{\alpha} \lambda \lambda \omega$ ) utilisé par Élien, qualifie ces animaux à la fois comme lanceurs de flèches et de venin. Le mot ios, « rare et poétique », désigne la flèche ${ }^{10}$ : par exemple, la première de celles qu'Apollon

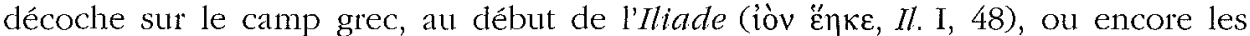
flèches ailées (ì̀̀ $\pi \tau \varepsilon p o ́ \varepsilon v \tau \alpha, I l . \mathrm{XX}, 68$ ) du dieu archer et les flèches qui tuent

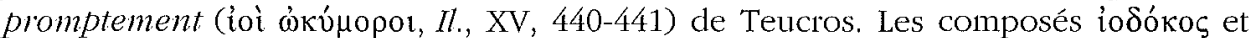

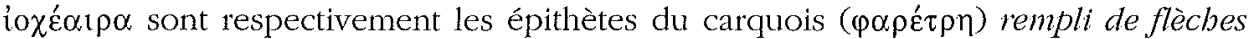
(Od. XXI, 11-12; Il. XV, 443-444, etc.) et d'Artémis qui répand des flèches, sagittaire (II. V, 53, etc.). Mais le mot iós désigne aussi le poison, le venin ${ }^{11}$ et chez Aristote (HA

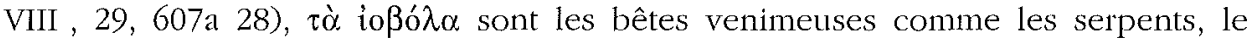
scorpion, le stellion.

Ainsi, la vipère, l'araignée «phalange » et le scorpion de Claros apparaissent comme de véritables «flèches au sol » auxquelles ne manque que le vol. Ce sont aussi les animaux terrestres probablement les plus redoutés, en pays grec, pour leurs piqûres et leurs morsures ${ }^{12}$. C'est sans doute pourquoi la venimeuse triade clarienne figure, aux côtés d'une main ouverte, d'une étoile de mer et d'un motif en spirale, sur une minuscule amulette en or découverte dans la « villa » d'Hagia Triada, en Crète ${ }^{13}$ (documents 1a et $1 \mathrm{~b}$ ). De même, parmi les quelques 130 sceaux du Minoen Moyen II (ca 1800 - ca 1700 avant J.-C.) retrouvés à Malia dans l'Atelier de Sceaux du Quartier Mu, plusieurs sont gravés d'une araignée ou d'un scorpion (document 2). Selon JeanClaude Poursat, "percés d'un trou qui permettait de les porter suspendus à des liens, en bracelet ou en collier, ils pouvaient sans doute servir d'amulettes (...) $\gg^{14}$.

10 Voir P. Chantraine, 2 iós, DELG I (1968) : « on pose "i $\sigma F-0$ - qui avec l'addition d'une voyelle thématique répond à skr. ísu-, av, išu-, 'flèche'. » A rapprocher du nom d'un artisan spécialisé de l'Inde ancienne que mentionne le Mabajjanaka-jātaka: l'isukara, chargé de redresser par chauffage les flèches faussées sur le champ de bataille. Voir J. Auborer, "L'arc et la flèche dans l'iconographie ancienne de l'Inde", Artibus Asiae 19 (1956), p. 184. Notons enfin que ótotós, très fréquent pour 'flèche', pourrait être lié étymologiquement à iós, 'flèche'. $C f . D E L G, s . v$.

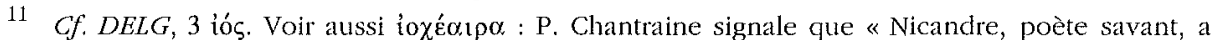
clétoumé le mot en le rattachant à łós 'poison' et l'a employé comme épithète de la vipère » (fi: 33) .

12 En témoigne un long passage de Galien dans Les endroits affectés - traité écrit vers la fin de sa vie sous le règne de Septime Sévère - qui, précisément, examine les effets des venins de la 'phalange' / tarentule, du scorpion et de la vipère, ainsi que certains traitements appropriés (VIII De loc. aff. III, 11, p. 194, 4-198, 5). Le médecin grec insiste, en particulier, sur l'extrême gravité des piqûres de scorpions, décrit les souffrances qu'elles entraînent et lá propagation du mal dans le corps (idem, p. 194, 4-197, 9). Voir P. Moraux, Galien de Pergame. Souvenirs d'un médecin, Paris, Les Belles Lettres, 1985 (Collection d'études anciennes), p. 49-51 et 54-55.

13 Pièce conservée au Musée d'Héraklion ( $n^{\circ} 141$, vitrine 101) dans la Salle VII qui expose des trouvailles provenant des mégarons, villas et grottes de Crète centrale (périodes néopalatiales et postpalatiales, $1700-1300$ av. J.-C.). Une araignée, un serpent et un scorpion, pinces ouvertes et queue relevée, sont représentés en relief autour d'un noyau de forme globulaire surmonté d'un anneau de suspension : chargé d'un pouvoir apotropaïque, ce pendentif-amulette était destiné à protéger celui qui le portait contre ces bêtes à venin. Pour une description de cette amulette, on peut se reporter à N. Platon, La civilisation égéenne, t. 2, Le bronze récent et la civilisation mycénienne, Paris, 1981, p. 58, ainsi qu'à I.A. Sakellarakis, Musée d'Héracleion. Guide illustré, Athènes, 1997 , p. 71.

14 J.-Cl. Poursat (avec la participation de M. Schmid), Guide de Malia au temps des premiers palais. Le quartier Mu, Athènes/Paris, 1992 (Sites et monuments, 8), p. 22. Voir aussi p. 23. Servir d'amulette n'était pas le seul usage de ces sceaux : J.-Cl. Poursat indique qu'ils « étaient avant tout 


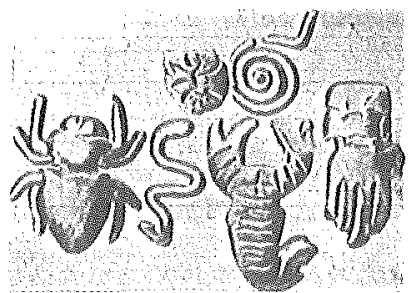

Doc. 1a

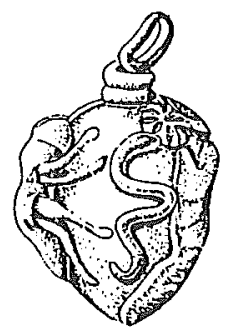

Doc. 1b

Amulette d'Hagia Triada

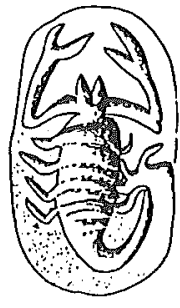

Doc. 2

Sceau au scorpion de Mallia

Parmi les trois iobola, ces « bêtes à flèches et à venin » que l'Archer interdit de sanctuaire à Claros, c'est précisément le scorpion qui va retenir notre attention : nous voudrions montrer ici combien sa présence aux côtés d'Apollon dans la tradition clarienne, revêt un intérêt tout particulier et n'a rien de fortuit.

Nous nous tournerons ensuite vers les frênes $\left(\mu \varepsilon \lambda i^{\prime} \alpha\right.$ l) de l'alsos clarien ${ }^{15}$. Au sein du panthéon, Apollon semble être le plus important propriétaire de bois sacrés. Grâce à Pausanias et à Strabon, notamment, nous savons que le dieu aime aussi le laurier à Didymes, le palmier à Chios, le cornouiller sur l'Ida de Troade et le cyprès à Daphnè, près d'Antioche ${ }^{16}$. Que signifie le frêne à Claros? Nous verrons que l'arbre dont l'Archer a recouvert son sanctuaire pour en chasser des animaux à la morsure ou à la piqûre venimeuses, des "animaux à flèches", est un arbre qui pique, un arbre en quelque sorte sagittaire et toxique qui entretient avec l'archerie apollinienne les rapports les plus étroits : le dieu qui peut envoyer le mal ou bien le repousser à l'aide de ses flèches, ne pouvait être mieux placé, au milieu de ses frênes, pour tenir à l'écart des animaux prompts à la décoche. Que l'alsos clarien soit une frênaie, me paraît donc aussi peu fortuit que la présence du scorpion dans la tradition dont Nicandre se fait l'écho.

À Claros, des caractères remarquables comme la situation au creux d'un « profond vallon », la présence d'une source, la présence des frênes, la forme de leurs feuilles, le grand nombre d'animaux piqueurs et venimeux, ont probablement ouvert à des observateurs aussi avisés du milieu biologique que l'étaient les Grecs, ce que Claude Lévi-Strauss appelle un « droit de suite »: il définit par cette expression le droit de « postuler que ces caractères visibles sont le signe de propriétés également singulières, mais cachées ${ }^{17}$. Il existe en effet des formes, des objets, des animaux, à

destinés à imprimer, sur des fragments d'argile crue, la marque personnelle de leur possesseur sous forme de scellés appliqués sur des vases, des portes de magasins, ou des documents » (p. 22). Sur ce système de scellés, voir p. 46-47.

15 Pausanias (VII, 5, 10) les mentionne quand il décrit les richesses naturelles de l'Ionie : « Sur

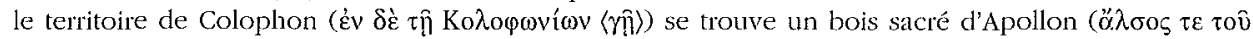

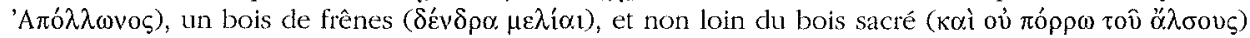

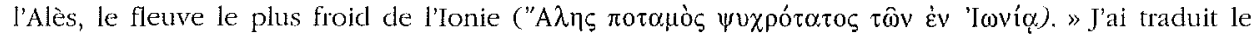
passage sur le texte grec de l'édition de la Loeb Classical Library (trad. W.H.S. Jones, réimpr, 1988 [1933]).

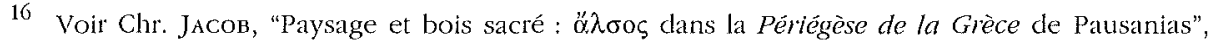
in Les bois sacrés, o.c. (n. 5), p. 40-41.

17 Cl. Lévi-Strauss, La pensée sanvage, Paris, 1962, p. 25. 
partir desquels l'imagination travaille et réagit. C'est la « logique concrète » ou « logique du concret » mise en lumière par le grand anthropologue ${ }^{18}:$ ne fonctionnant pas d'une façon très rationnelle, elle met en jeu l'intuition, la perception sensible, l'imaginaire, avec l'établissement d'associations, de connections, de systèmes de correspondances qui peuvent déconcerter un esprit scientifique contemporain. On peut considérer que cette « logique du concret » opère en arrière-plan des récits d'Élien et de Nicandre à l'origine de notre étude. Aussi, quand l'historien essaie de "décortiquer» la tradition clarienne rapportée par ces auteurs, d'en révéler les structures sous-jacentes, est-il amené à « tirer sur toutes sortes de ficelles ${ }^{19}$ qui tissent un complexe foisonnant, aux nombreuses facettes, mais articulé et cohérent, organisé autour d'Apollon et de la flèche qu'il décoche. Ce faisant, l'historien propose peut-être une lecture plus complète et plus explicite que celle qu'un Grec aurait pu faire. Quand il tente dans son travail de reconstruction, de mettre au jour le système de relation caché qui gouverne la tradition clarienne, sans doute se heurte-t-il à une límite familière aux ethnologues et que Philippe Descola identifie clairement de la façon suivante : «Ce que les ethnologues appellent un système de représentation n'est généralement systématique que pour l'observateur qui le reconstruit et en dégage les structures; la logique sous-jacente isolée par l'analyse savante affleure en effet rarement à la conscience des membres de la culture étudiée, qui ne peuvent pas plus la formuler qu'un jeune enfant n'est capable de traduire en règles la grammaire d'une langue dont il a pourtant la maîtrise ${ }^{20}$.

Le chemin que nous allons parcourir, à l'ombre des frênes et à l'abri des scorpions, dans le sanctuaire d'Apollon à Claros, peut alors s'apparenter à la démarche ethnologique telle que la définit cet auteur : un « exercice de formalisation », une « entreprise d'explication de l'implicite ${ }^{21}$.

Pour commencer, les scorpions de Claros. Et d'abord, quels scorpions? Parmi la vingtaine d'espèces de scorpions réputées très dangereuses, on peut en retenir trois, familières des bords de l'Égée et susceptibles d'avoir fréquenté le site de Claros dans l'Antiquité $^{22}$ : le scorpion gibbeux (Mesobuthus gibbosus), une espèce proche, Iurus dufoureius et le scorpion des Carpathes (Euscorpius carpathicus) ${ }^{23}$. Ce sont des animaux dont le voisinage est périlleux, le scorpion gibbeux notamment. Celui-ci se rencontre en Albanie, Grèce et Turquie, jusqu'à Chypre, et se distingue par la toxicité de son venin : c'est « le plus dangereux des scorpions européens ${ }^{24}$. Il appartient à la famille des Butbidae qui - comme le signalent à plusieurs reprises les spécialistes -

18 Ibid., p. 50, 88, 193 et 346.

19 J'emprunte l'expression à Jean-Plerre Vernant dans son introduction aux actes du Colloque interdisciplinaire "La Grèce pour penser l'avenir ", tenu à Paris-Sorbonne et au Palais du Luxembourg, du 2 au 4 déc. 1996, Paris, L'Harmattan, 2000 (Collection L'Homme et la Société), p. 16.

20 Ph. Descola, Les lances du crépuscule. Relations jivaros. Haute-Amazonie, Paris, Plon, 1993 (Terre Humaine), p. 165-166.

21 Ibid., p. 166.

22 Cf. DELG, бкорлі́⿴\zh11 : l'étymologie du mot est 'ignorée' mais son origine méditerranéenne est 'plausible'.

23 Pour une présentation de ces espèces, on peut se reporter à G. Dupré, N. Lambert et Ph. Gérard, Les Scompions. Biologie, élevage, Paris, 1998 (Les nouveaux animaux de compagnie). Très utile aussi, R. Stockmann, M. Goypfon, "V. Les Scorpions", in M. Goy'fFon et J. Heurtault (éds), La fonction venimeuse, Paris, Masson, 1995 (Biodiversite, Série Sciences naturelles), p. 88-100.

24 Dupré et alii, o.c. (n. 23), p. 61. 
comprend les espèces les plus dangereuses, et parfois mortelles pour l'homme ${ }^{25}$. Le deuxième, de la famille des Iuridae, est originaire de Grèce et de Turquie. C'est «le plus grand scorpion d'Europe avec une taille de plus de $10 \mathrm{~cm} »^{26}$. Quant au troisième, de la famille des Chactidae, il est présent dans les Balkans et en Turquie ${ }^{27}$. Les piqûres de ces deux dernières espèces sont douloureuses, mais sans conséquence pour l'homme. Cette tentative d'identification permet de saisir avec plus d'acuité la réalité de la menace que cet animal pouvait représenter, au point de motiver la garde que montaient Apollon et ses frênes pour l'écarter du sanctuaire clarien.

Ceci posé, qu'est-ce qu'un scorpion pour les Grecs? Un passage des Parties des

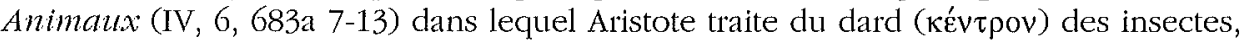
dresse le portrait d'un scorpion très militaire et fait pour les champs de bataille :

Ceux dont le dard est par derrière s'en servent comme d'une arme, parce qu'ils

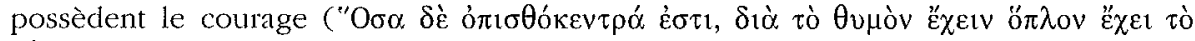

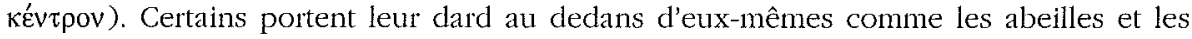
guêpes, parce que ces insectes sont ailés : si leur dard était léger et externe il s'abîmerait facilement; et s'il était proéminent comme celui des scorpions, il les alourdirait

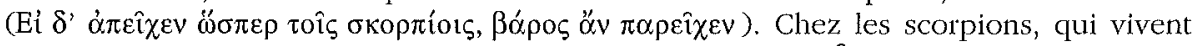

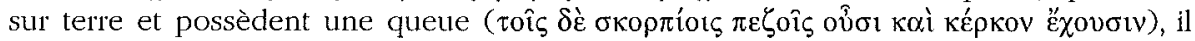
est nécessaire que le dard soit sur cette queue, sinon il ne servirait à rien pour le

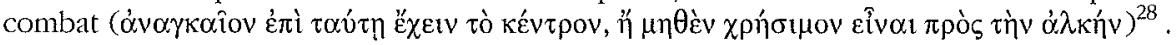

Il faut s'arrêter sur le vocabulaire utilisé ici. Le terme ö $\pi \lambda$ ov tout d'abord, surtout au pluriel, signifie "instruments », mais c'est avant tout un terme désignant les « armes » du guerrier ${ }^{29}$. Son emploi pour désigner le dard proéminent dont est naturellement équipé le scorpion, fait de cet animal un guerrier équipé d'une « arme » ${ }^{30}$. Pourvu de $\theta v \mu o ́ s$, le scorpion d'Aristote est aussi fortement armé d'ardeur belliqueuse : après le sens «âme, cour" », « en tant que principe de la vie », le mot signifie aussi « ardeur, courage, siège des sentiments et notamment de la colère ${ }^{31}$. Armé, agressif, le scorpion est aussi un combattant à pied. Pour le distinguer du scorpion de metr $^{32}$, Aristote le qualifie de $\pi \varepsilon \zeta o_{\zeta}$, littéralement qui va à pied. Cet adjectif, courant chez Aristote, qualifie le scorpion comme animal qui vit ou va sur terre. Il appartient aussi au vocabulaire militaire et désigne le guerrier combattant à pied -

\footnotetext{
25 Dupré et alit, o.c. (n. 23), p. 7, 19-21, 58. Voir aussi Stockmann-GoyFFon, l.c. (n. 23), p. 93, 96-99.

26 Dupré et alli, o.c. (n. 23), p. 66.

27 Dupré et alii, o.c. (n. 23), p. 21 et 62.

28 Texte établi et traduit par P. Louis, Paris, Les Belles Lettres, 1990 [1957].

29 Le mot a le sens technique de 'bouclier' de l'hoplite - ainsi chez Hérodote - et désigne la pesante panoplie du guerrier lourd, dans l'Anabase de Xénophon, par ex. Il signifie aussi 'arme' en général, y compris en parlant d'arc et de flèches. C'est le cas dans le débat de l'Héraclès d'Euripide qui illustre la vieille opposition entre le guerrier à l'arc et le guerrier lourd, et qui développe les

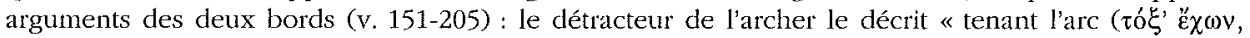

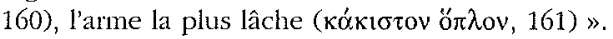

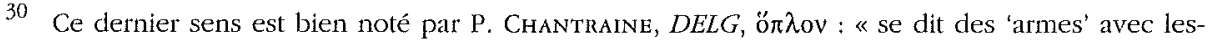
quelles un animal se défend ». Aristote souligne la grande taille de ce dard ( $H A$ IV, 7 532a 16-17).

$31 D E L G, \theta v \mu o ́ s$ : «toute la dérivation se rapporte à la notion de colère, d'humeur, etc., "

32 Il s'agit de la scorpène, vulgairement appelée 'rascasse', poisson toxique de l'ordre des Scorpaénidés.
} 
voir le français «piéton »/ « piétaille »- et comme substantif collectif, l'infanterie ${ }^{33}$. Les termes utilisés dans ce passage font du scorpion un fantassin à l'humeur comba-

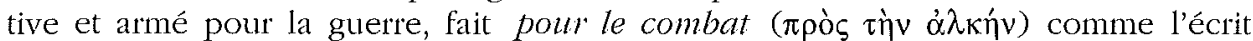
Aristote $^{34}$. Or, le combattant ainsi décrit dans les Parties des Animaux est bien un archer.

C'est ce que suggère fortement le marticbore, créature fantastique de l'Inde, d'après la description qu'en fait Aristote à l'intérieur d'un long développement sur les dents des animaux (HA II, 1, 501a, 24 sq.):

Aucun de ces genres d'animaux ne possède à la même mâchoire une double rangée de dents. Il y a pourtant un animal de cette sorte, s'il faut en croire Ctésias. Cet auteur raconte qu'il existe aux Indes une bête sauvage du nom de martichore, qui possède à chaque mâchoire une triple rangée de dents; sa taille est celle du lion, il est velu comme lui, et ses pieds sont pareils aux siens, il a le visage et les oreilles d'un homme, les yeux bleus et le corps d'un rouge de cinabre; sa queue ressemble à celle du scorpion terrestre (

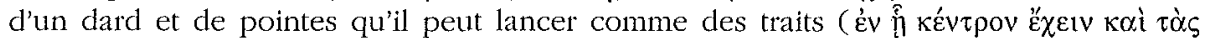
$\dot{\alpha} \pi \circ \varphi v \alpha \dot{\alpha} \delta \alpha \varsigma \dot{\alpha} \pi \alpha \kappa o v \tau i \zeta \zeta \varepsilon(v)$; sa voix est pareille au son de la flûte et à celui de la trompette à la fois; sa vitesse à la course atteint celle du cerf; enfin, il est cruel et mange la chair humaine ${ }^{35}$.

C'est le tigre qui se cache derrière cet animal fantastique. Ctésias de Cnide, médecin et auteur des Indica, recueil de mirabilia sur l'Inde ${ }^{36}$, dont s'inspire ici Aristote, en fait une description déformée par la terreur que causait cet animal mangeur d'hommes ${ }^{37}$. Sous le coup de la crainte que leur inspirait le puissant carnivore, influencés aussi par sa provenance - l'Inde étant pour eux la terre de tous les émerveillements - les Grecs ont dressé le portrait chimérique d'un monstre fabuleux. Or,

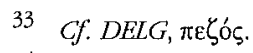

34 Il n'est pas inutile de remarquer que les spécialistes de l'éthologie s'en tiennent aujourd'hui à ce que les Grecs avaient observé : c'est à l'article 'combat' de son Dictionnaire du comportement animal, Paris, 1990 [Oxford University Press, 1981], p. 186) que D. McFarland traite du scorpion. Voir aussi J.-H. FABRE, Souvenirs entomologiques. Eitudes sur l'instinct et les mœurs des insectes II, Paris, 1989 [1925] : l'auteur décrit un véritable combattant, toujours prêt à se défendre et à attaquer (p. 260, 793, 799, 802, 810-811, 822). Observateur passionné, il organise des 'batailles entomologiques', entre le scorpion et des insectes, pour juger de la violence du venin. De véritables duels dont le scorpion sort invariablement vainqueur. $C f$. p. 810-821.

35 Texte traduit par J. Tricot, Paris, Vrin, $1987^{2}$. P. Louis donne une traduction très voisine (Paris, Les Belles Lettres, 1964).

36 Sur Ctésias et son œuvre historico-romanesque, voir l'introduction de $J$. Auberger qui a traduit les Histoires de l'Orient de Ctésias (Paris, Les Belles Lettres, 1991, p. 1-14) et P. Briant, Histoire de l'Empire perse de Cyrus à Alexandre, Paris, Fayard, 1996, p. 14, 276. Pour une présentation plus 'médicale', voir J. Jounnna, Hippocrate, Paris, Fayard, 1992, p. 40, 76-80, 226. Membre de la branche des Asclépiades de Cnide, ce jeune parent d'Hippocrate a peut-être été fait prisonnier de guerre par les Perses et devint le médecin du roi Artaxerxès II. Une fois revenu en Grèce, vers 398-397, il publia ses Persica et ses Indica. C'est à la cour achéménide, où il était un témoin privilégié des relations entre Perses et Indiens, qu'il puisa la matière de son traité sur l'Inde, que nous connaissons surtout par la Bibliotbèque (Codex 72) de Photius.

37 Pausanias reconnaît le tigre (IX, 21, 4). Voir les notes ad loc, de P. Louis et J. Tricot ainsi que la préface de Ch. Malamoud à Auberger, o.c. (n. 36), p. xiv et la note 11, p. 167 : "'Martichora' vient du perse Martiya 'homme' + khwar 'manger' = 'Mangeuse d'hommes', » 
cet animal hybride a une queue qui tient du scorpion et c'est précisément cette ressemblance qui fait du martichore une espèce d'archer ou de lanceur de javelot.

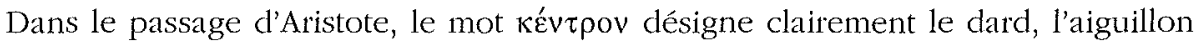
venimeux qui termine l'abdomen mobile du scorpion et qu'il projette pour piquer sa proie. Cette détente vers l'avant explique sans doute la croyance selon laquelle la queue de scorpion du martichore était armée de pointes qu'elle pouvait propulser au

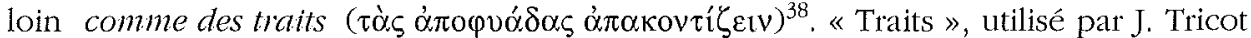
et $\mathrm{P}$. Louis dans leurs traductions, est un terme générique qui désigne tout projectile lancé par une arme de jet - javelot, flèche, etc. - mais le dénominatif $\dot{\alpha} \pi$ - $\dot{\alpha} \kappa o v \tau i \zeta \zeta \omega$ signifie précisément lancer des javelines, des javelots ${ }^{39}$. De fait, le geste technique caractéristique de l'aconte qui projette avec force son bras d'arrière en avant pour lancer son javelot, répond parfaitement au mouvement rapide de la queue du scorpion $^{40}$. Mais l'image de l'archer peut tout aussi bien sous-tendre la description d'Aristote : la rapidité de la détente quand le scorpion darde son aiguillon, évoque le moment de la décoche, avec la brusque détente des bras de l'arc vers l'avant et le jaillissement de la flèche ${ }^{41}$. L'aiguillon presque toujours brandi vers l'avant, au bout d'une queue largement recourbée, le scorpion semble être en quelque sorte, tout à la fois l'arc arrondi en position armée (document 3a), la flèche qui s'élance et l'archer qui tire ${ }^{42}$. L'affinité entre le scorpion et l'archer sort encore renforcée du texte de Ctésias sur le martichore tel que l'a utilisé Élien, cinq siècles et demi après Aristote. Le portrait qu'il fait de cette chimère indienne est très proche de celui du maître du Lycée, mais plus détaillé en ce qui concerne la queue de scorpion $(N A I V, 21)$ :

À l'extrémité de sa queue est fixé un dard de scorpion ( бкорлі́ou Kévтроv) qui peut bien dépasser une coudée, et sa queue porte une rangée de piquants de chaque côté. Le bout de sa queue pique mortellement l'homme qui se présente, et le tue instantanément ( $\kappa \alpha i ̀ ~ \delta 1 \varepsilon ́(\varphi \theta \varepsilon ı \rho \varepsilon \pi \alpha \rho \alpha \chi \rho \hat{\mu} \mu \alpha$ ). Si quelqu'un le pour-

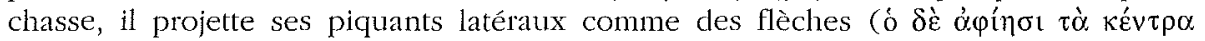

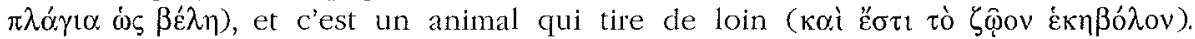

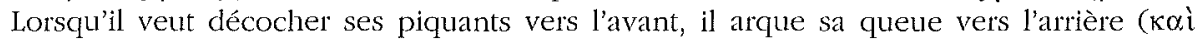

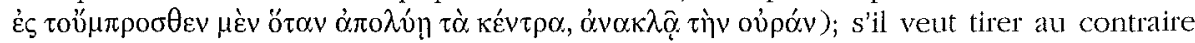

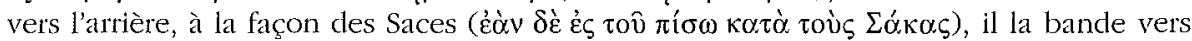

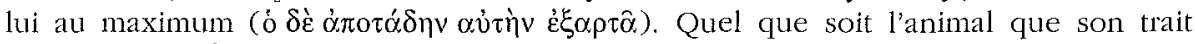
atteint ( ‘ò $\beta \lambda \eta \theta \dot{v} v$ [litt. ce qui a été lancél), il le tue, mis à part l'éléphant, qui n'en

$38 \alpha \alpha \pi$ a $v o ̛ ́ \delta \alpha \zeta$ désigne des appendices, mais le sens de 'pointes' donné par J. Tricot et P. Louis, "s'éclaire si l'on compare avec le texte de Ctésias cité par Photius ». Cf. Lous, n. 3 ad loc..

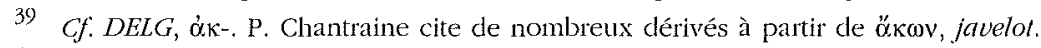

40 La queue recourbée et relevée par-dessus le dos évoque la lance brandie en l'air prête à frapper.

41 La simple observation de l'animal suffit, en effet, à suggérer l'image de l'archer: pour décrire le scorpion sur ses gardes face à une scolopendre, Fabre, o.c. (n. 34), parle de façon significative de «l'arc de sa queue tendu » (p. 820) et à propos de cette queue toujours recourbée, il précise que «bien rarement il la traîne, débandée en ligne droite » (p. 794). Le terme 'débandé' qui dit l'absence d'agressivité du scorpion, l'état de repos de sa queue, évoque fortement le vocabulaire de l'arc: en terme d'archerie, débander l'arc signifie le clésencorder, enlever la corde. L'arc est alors inapte au tir, en position de repos.

42 Une telle comparaison en trois temps se retrouve chez Aristote, et de façon très explicite, à propos du dauphin, pensionnaire privilégié du bestiaire apollinien : l'animal prend son élan comme l'archer qui bloque sa respiration pour viser, puis s'arrondit comme un arc, et enfin fuse comme une flèche (HA IX, 48, 631a $23 s q$ ). 


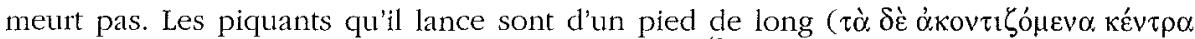

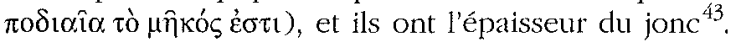

Avec sa queue de scorpion hérissée de piquants, le martichore est une vivante

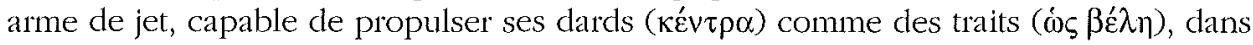
toutes les directions, Le terme $\beta \varepsilon ́ \lambda$ os désigne tout projectile, tout trait lancé que seul le contexte permet d'identifier comme javelot ${ }^{44}$, flèche ${ }^{45}$ ou autre. Mais, même si les

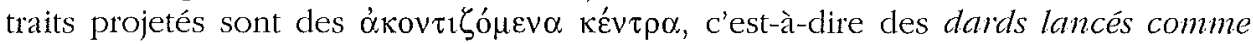

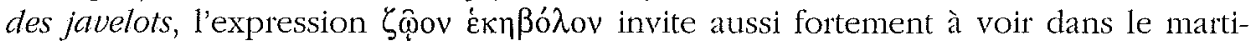

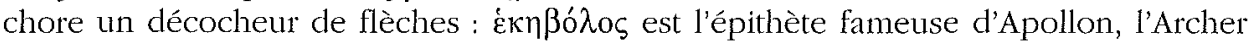
qui lance au loin, qui frappe de loin avec ses flèches sans manquer le but ${ }^{46}$. Au premier chant de l'Iliade par exemple, c'est cette épithète qui qualifie (v. 14, 21) ou désigne seule $(v .96,110)^{47}$ le dieu à l'arc décochant ses flèches « pestilentielles » sur le camp grec ${ }^{48}$. Véritable « animal de jet », tout comme l'arc est un « instrument de jet », le martichore békebolos décoche avec sa queue de scorpion à longue portée des flèches qui frappent loin et juste, comme celles d'Apollon au tir puissant et infaillible: Il faut alors noter que Pausanias assimile sans ambiguïté le martichore, avec sa queue munie de dards, à un archer (IX, 21, 4) :

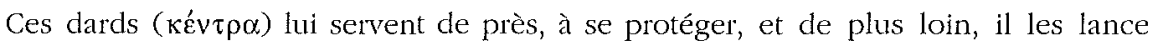

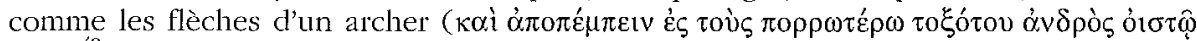
i $\sigma \circ)^{49}$.

À travers les textes d'Aristote, d'Élien et de Pausanias, le scorpion apparaît comme un belliqueux lanceur de traits, plus sagittaire qu'aconte, qui « décoche » véritablement son dard à la manière d'un archer et qui, tout autant qu'Apollon Hékebolos ou Hékatebolos, «lance ses flèches au loin ».

Cette interprétation trouve l'appui d'un argument supplémentaire dans le vocabulaire du machinisme militaire : en conformité parfaite avec la queue du martichore, le nom de «scorpion » désigne aussi une catapulte à flèches. On considère traditionnellement, à la suite de Diodore de Sicile, que les premières catapultes furent inventées à Syracuse, aux environs de 400 av. J.-C., par les ingénieurs mécaniciens du tyran Denys l'Ancien en guerre contre les Carthaginois ${ }^{50}$. Y. Garlan décrit ainsi ces vérita-

43 Texte traduit par Zucker, o.c. I (n. 2). On peut se reporter aux traductions de Scholfield, o.c. (n. 4), vol. I, 1971 [1958], et d'Auberger, o.c. (n. 36), p. 118.

44 Ainsi la lance avec laquelle Ulysse tue Démocoon (Il. IV, 498).

45 Ainsi les flèches par lesquelles Apollon procure une mort douce (II. XXIV, 759; Od. III, 280).

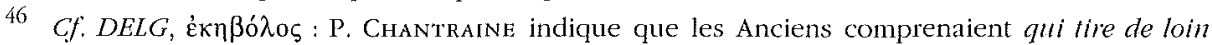

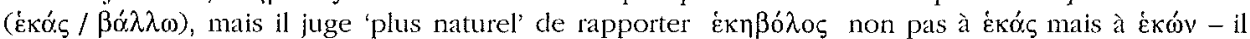

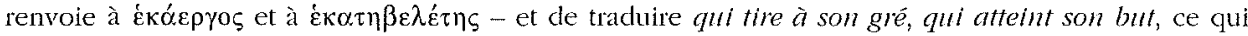
n'empêche pas que « le rapprochement avec ékós par étymologie populaire est probable ».

47 Voir aussi l'Hymne ps.-hom. à Apollon, 45 et l'Hymne ps.hom. à Hermès, 218, 509, 522

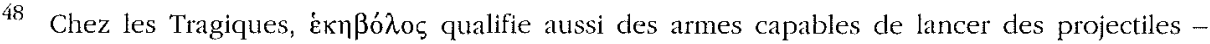
arcs, frondes - ou les mains de Zeus et dans la prose hellénistique, des guerriers lanceurs de traits ou les traits eux-mêmes. Cf. DELG et le L-S-J, s.v.

49 J'ai traduit le passage sur le texte de la Loeb Classical Library (traduction de W.H.S. Jones, réimpr. 1995 [1935]).

50 Comme y invite P. Briant, “À propos du boulet de Phocée”, REA 96 (1994), p. 111-114 et o.c. (n. 36), p. 783,933 , il semble que l'étude des boulets perses découverts à Phocée et à Paleapaphos 
bles pièces d'artillerie : «Il s'agissait d'engins anti-personnel, usant de flèches comme projectiles : il est possible que leur système propulseur ait été fondé, non plus sur le principe de l'arc, mais déjà, comme il le sera incontestablement dans la seconde moitié du Iv ${ }^{\mathrm{e}}$ siècle, sur l'élasticité de tendons, cheveux ou crins soumis à un effort de tension. À ces « oxybèles » s'ajoutèrent, à partir du siège de Tyr par Alexandre en 332, des catapultes à boulets portant le nom de «pétroboles » ou "lithoboles ». Les uns et les autres atteignirent leur maximum de puissance dès la fin du $\mathrm{IV}^{\mathrm{e}}$ siècle... $»^{51}$. Selon leur calibre, les oxybèles ${ }^{52}$ pouvaient propulser des flèches mesurant de $70 \mathrm{~cm}-1$ la longueur moyenne d'une flèche d'arc - à $2 \mathrm{~m}$, et $\mathrm{Y}$. Garlan indique qu'« aux oxybèles de faible calibre paraît avoir été réservé le nom de «scorpions $»^{53}$. Ce type de catapulte a donc connu deux modes de propulsion au cours du $\mathrm{IV}^{\mathrm{e}}$ siècle. Le premier était fondé sur le principe de la tension : un arc d'une très grande force, «composite » et "réflexe ${ }^{54}$ (document $3 \mathrm{~b}$ ), était monté sur un affût et s'armait au moyen d'un treuil et de leviers (document 4a). Le second, dès le milieu du Iv ${ }^{\mathrm{e}}$ siècle, peut-être avant, eut recours à la force de la torsion: deux écheveaux ou faisceaux de fibres élastiques, puissamment torsadées, servaient de ressorts et propulsaient deux bras de bois, mobiles, engagés, à une extrémité, dans les deux faisceaux de câbles et qui recevaient, à l'autre bout, la corde archère (document 4b). Machine « à tension » ou « à torsion », le scorpion n'en reste pas moins un arc mécanique, un engin à décocher des flèches, pensé et bâti sur le modèle de l'arc proprement dit. On s'explique maintenant mieux l'homonymie entre cette machine de jet et le scorpion : les Grecs ont baptisé une petite catapulte à flèches du nom d'un animal qui frappe ses proies sur le modèle de l'archer, d'un « animal-archer » décochant lui aussi ses flèches.

D'autres éléments viennent plaider en faveur d'une étroite affinité entre le scorpion et l'archer : il est significatif que l'imaginaire hellénique ait pu associer le scorpion et la Scythie. Considérons le passage de l'Histoire des Animaux dans lequel Aristote traite de l'influence des lieux sur certaines bêtes venimeuses (VIII, 29, 607a 13-20) :

En ce qui concerne les morsures ( $\delta \eta ́ \gamma \mu \alpha \tau \alpha$ ) des bêtes, les divers pays présentent également de grandes différences : ainsi près de Pharos et en d'autres lieux les

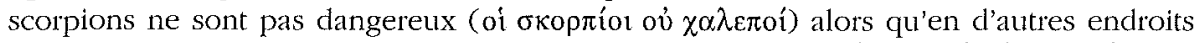

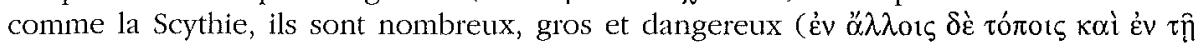

conduit à remonter d'un siècle et demi au moins l'apparition des premières machines de jet et à déplacer leur invention de la Sicile vers l'empire achéménide.

51 Y. Garlan, "La poliorcétique", Dossiers d'Arcbéologie 172 (juin 1992), p. 31-32. Voir, du même auteur, Guerre et économle en Grèce ancienne, Paris, 1989, p. 124.

52 C'est-à-dire au trait acêré, à la pointe aiguë. L'adjectif qualifie la flèche avec laquelle

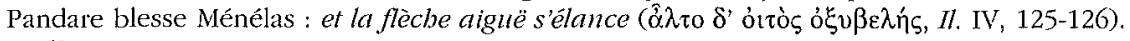

53 L.c. (n. 51), p. 32. E.W. Marsden (Greek and Roman Artillery. Historical Development, Oxford, Clarendon Press, 1999 [1969], p. 188) relève aussi que les plus petites des catapultes à flèches sont appelées 'scorpions' en grec comme en latin. Dans leur traduction commentée du Livre $\mathrm{X}$ du De Architectura de Vitruve, L. Callebat et Ph. Fleury (Paris, Les Belles Lettres, 1986) notent que si les termes scorpio et catapulta désignent un même type de 'lanceur de flèches', le premier est «plus particulièrement appliqué à de petites catapultes » (n. 2, p. 199 à X, 10, 1).

54 'Composite', parce que fait de plusieurs matériaux - bois, tendons, corne - dont les qualités mécaniques se complétaient. 'Réflexe', parce que pour bander et armer un tel arc, il fallait inverser la courbure naturelle de ses bras - mouvement caractéristique rendu par l'épithète homérique palintonos - qui retrouvaient leur position initiale une fois l'arc débandé. 


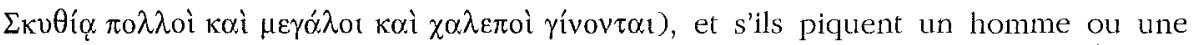

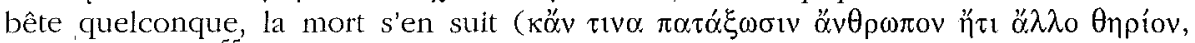

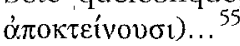

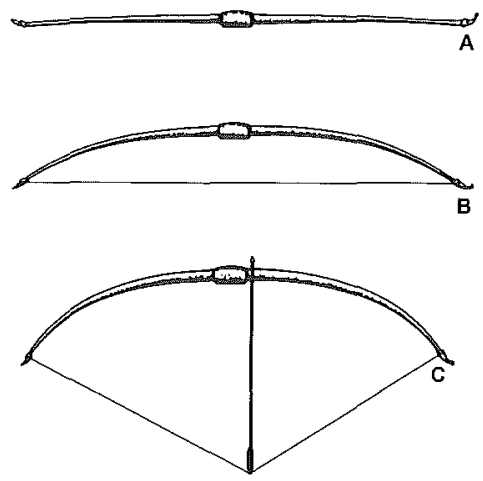

Doc. $3 a$

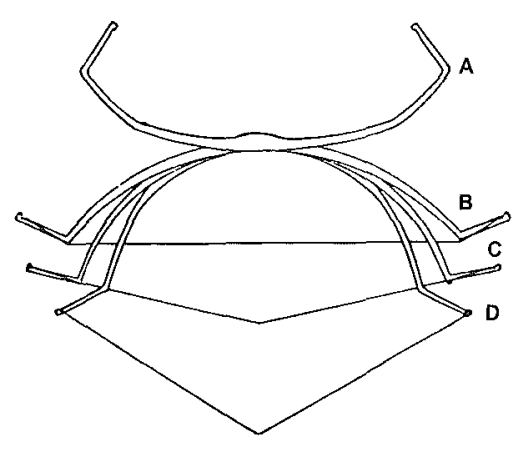

Doc. $3 \mathbf{b}$

Les trois positions de l'arc : $A$ : au repos; $B$ : bandê; $C$-D : armé

Ainsi, selon les Grecs, c'est chez les Scythes, dans un pays réputé pour ses archers, que prospéraient les plus gros et les plus dangereux des scorpions ${ }^{56}$. Au reste, les Scythes étaient, comme les scorpions, «venimeux» et particulièrement «toxiques », au sens étymologique du terme ${ }^{57}$, considérés comme des experts dans la préparation des poisons de flèches ${ }^{58}$. N'est-ce pas alors parce qu'ils associaient étroitement le scorpion et l'archer scythe, que les Grecs plaçaient les plus dangereux des scorpions en Scythie? C'était traduire dans l'espace la grande affinité qui leur semblait réunir l'animal et l'archer ${ }^{59}$.

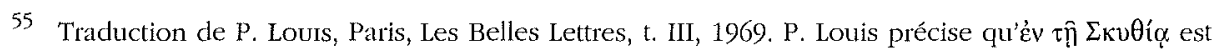
« la leçon des meilleurs manuscrits » (n. 2 ad loc.). C'est celle que confirme Pline dans le plus long développement qu'il consacre aux scorpions (HN XI, 90).

56 Cette croyance trouve un certain écho dans la carte de la répartition actuelle des scorpions établie par R. Stockmann et M. Goyfron, l.c. (n. 23), p. 94 : dans les étendues autrefois parcourues par les Scythes orientaux, les scorpions représentent aujourd'hui une réelle menace.

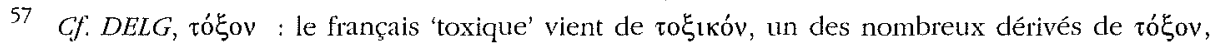
l'arc, qui désigne le 'poison dont on enduit les flèches' .

58 L'auteur aristotélicien du traité Des faits surprenants, 141 (845a), décrit la préparation du $\Sigma \kappa v \theta$ кòv $\varphi \alpha ́ p \mu \alpha \kappa o v$, ce 'poison scythique', mortel mélange de jus de serpents pourris et de sang humain décomposé. On pourra se reporter au texte grec et à la traduction anglaise de W.S. Hett dans la Loeb Classical Library XIV, réimpr. 1980 [1936]. Recette comparable et tout aussi irrémédiable chez Pline (INN XI, 279) et chez Élien (NA IX, 15).

59 On retrouve une zoogéographie symbolique de même nature à propos de la chouette: Pline $(H N \mathrm{X}, 76)$, Élien $(N A \mathrm{~V}, 2)$ et Solin $(\mathrm{XI}, 14)$ rapportent que la chouette ne vit pas en Crète et que, si on l'y importe, elle meurt. Il s'agit à l'évidence d'une légende, la zoologie moderne ignorant cette 'allergie' à la Crète. L'explication de cette incompatibilité entre l'oiseau et l'île pourrait se trouver dans l'association entre la chouette et Athéna. L'oiseau emblématique d'Athéna, la déesse hoplite, équipée de la lance et du bouclier, ne peut supporter la Crète, ce pays d'archers, étranger aux hoplites. Le parallèle me semble frappant entre l'animal-hoplite, la chouette, qui ne peut pas vivre dans la crète des arcs et des flèches et l'animal-archer, le scorpion, qui s'épanouit particulièrement dans la très 'toxophile' Scythie. 


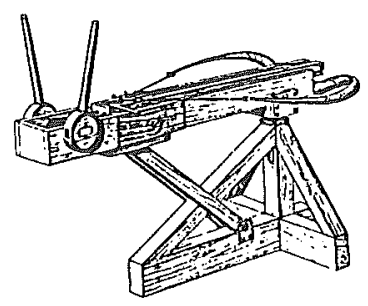

Doc. 4 a

Oxybèle «à tension»

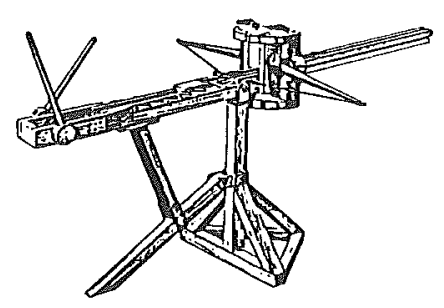

Doc. $4 b$

Oxybèle « à torsion»

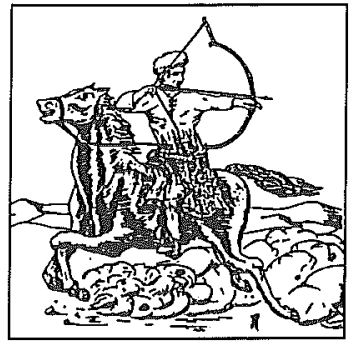

Doc. 5

La «flèche du Parthe »

Cette hypothèse me paraît confortée par un court passage du texte d'Élien sur le martichore qui témoigne d'une certaine complicité entre le scorpion et l'archer scythe. On se souvient que pour décocher son dard/trait vers l'arvière, le marti-

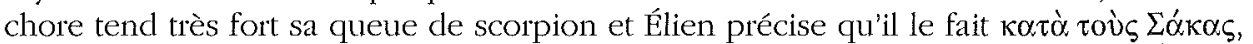
comme les Saces et non pas contre les Saces, comme le comprend J. Auberger ${ }^{60}$. Les Sakai, ou Saces, forment la famille des Scythes d'Asie centrale, nomades ou seminomades iranophones, qui vivent dans les steppes au-delà de la Sogdiane ${ }^{61}$. Ces Sakai sont avant tout connus comme des archers. Dans la Cyropédie (V, 3, 24),

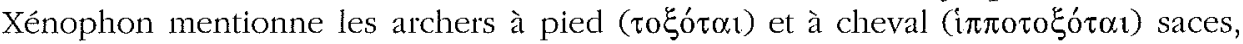
alliés de Cyrus contre les Assyriens. Hérodote rapporte que dans l'armée de Xerxès, its

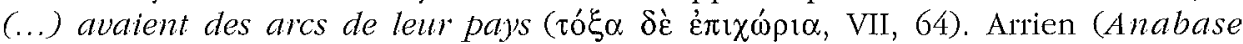
d'Alexandre le Grand III, 8, 3) signale les $i \pi \pi 0 \tau 0 \xi o ́ \tau \alpha 1$ saces dans l'armée perse qu'Alexandre écrase à Gaugamèles en 331. Revenons au texte d'Élien. Avec sa queue de scorpion, le martichore décoche son dard vers l'arrière ( $\tau$ ôิ $\pi i ́ \sigma \omega$ ). Comment ne pas reconnaître ici une allusion à la fameuse technique de la «flèche du Parthe »? Il s'agissait pour l'archer à cheval qui venait de charger l'ennemi aut galop, de continuer à décocher ses flèches alors même qu'il avait fait demi-tour et qu'il s'éloignait (document 5) : il devait pour cela pivoter sur sa monture, dans une manouvre demandant beaucoup d'adresse. Cette technique porte le nom d'un peuple d'origine scythe du sud-est de la mer Caspienne, mais elle fut utilisée par tous les archers nomades d'Asie. Si le martichore peut être comparé à l'archer scythe ou sace dans ce que sa science de l'arc a de plus remarquable, c'est bien que le scorpion qui prête sa queue au martichore, était considéré comme un archer et par là aisément identifiable à un Scythe.

60 O.c. (n. 36), p. 118. Grammaticalement défendable, la lecture contre me semble moins pertinente que celle retenue par Scholfield, o.c. (n. 43), et Zucker, qui traduisent respectivement as the Sacae do et à la façon des Saces.

61 Sur les Saces et leur 'identité' scythe, voir Briant, o.c. (n. 36), p. 49-50, 188-189, 553-556 et I. LebEdYNSKy, Les Scytbes, La civilisation des steppes (VIT - IIf siècles av. J.-C.), Errance, 2001, p. 7, 50-51, 54-57. Hérodote les présente comme une force d'élite de l'armée achéménide : à Marathon, les Saces forment, aux côtés des Perses, le centre de l'infanterie (VI, 113), alors qu'à Platées, la cavalerie sace se distingue par sa valeur (IX, 71). L'historien grec ne les distingue pas des Scythes quand il décrit leur contingent dans l'armée de Xerxès : « les Saces, - c'est-à-dire les Scythes - ... » ( $\Sigma$ ákoı $\delta \dot{\varepsilon}$ oi $\Sigma k u ́ \theta \alpha 1$, VII, 64). Voir aussi l'Histolre d'Alexandre (L'Anabase d'Alexandre le Grand III, 8, 3) d'Arrien. 
Le portrait qui se dessine d'un scorpion-archer, en quelque sorte "scythisé », trouve l'appui d'un récit d'apparence anecdotique relaté par Arrien dans lequel on retrouve le scorpion comme engin à flèches. À l'automne 329, au cours des difficiles opérations pour soumettre la Sogdiane, Alexandre se heurte à des Scythes orientaux : les cavaliers à l'arc saces le provoquent juste de l'autre côté de l'Iaxartes, l'actuel SyrDaria. Arrien rapporte qu'Alexandre fait donner l'artillerie contre eux, par-dessus le fleuve, avant de faire traverser son armée (IV , 4, 4):

Et, dès que les peaux furent prêtes pour le passage et que les troupes, armées de pied en cap, se furent placées au bord du fleuve, les machines ( $\mu \eta \chi \alpha v \alpha i ́)$, à un signal convenu, criblèrent de traits les Scythes qui passaient à cheval le long de la rive ( $\alpha i$ í $\tau \varepsilon$

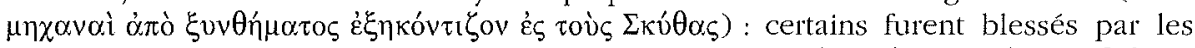
projectiles et même l'un d'entre eux, touché par un trait (غ̇лı qui avait traversé complètement son bouclier et sa cuirasse, tomba de cheval; les autres furent frappés de stupeur devant ces projectiles qui les atteignaient de si loin

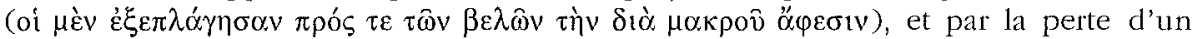
brave à eux, et ils s'éloignèrent un peu de la rive. Voyant que ces volées de traits avaient jeté le trouble parmi eux, Alexandre, au son des trompettes, commença à traverser le fleuve... ${ }^{62}$.

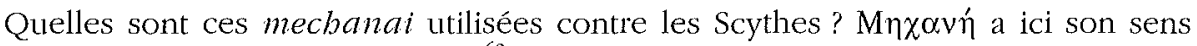
particulier de machine de guerte ${ }^{63}$ et le contexte indique clairement que les traits $(\beta \varepsilon ́ \lambda \eta)$ propulsés sont des flèches : il s'agit donc très vraisemblablement de scorpions mis en batterie sur la rive ${ }^{64}$. Les mechanai appelées scorpions par analogie avec un animal réputé décocher son clard comme une flèche, montrent leur efficacité dans une région renommée précisément pour ses très dangereux scorpions et pour ses archers aux flèches empoisonnées. Frappant avec son dard comme le scorpion, venimeux comme lui, l'archer scythe, véritable «scorpion à cheval » ${ }^{65}$, est un mal que les Grecs neutralisent par un remède de même nature que lui : une catapulte à flèches, c'est-à-dire un autre scorpion. Certes il s'agit là de liaisons symboliques et le simple souci d'efficacité tactique commandait de frapper les Scythes de loin, grâce à l'artillerie. Toutefois on ne peut ignorer les rapprochements et les correspondances mis en lumière plus haut et qui s'articulent autour du scorpion. J'en viendrais volontiers à penser que dans l'univers mental des Grecs, utiliser ces engins à flèches contre les archers scythes c'est aussi, en quelque sorte, utiliser le scorpion comme antidote contre lui-même, pour mieux lutter contre sa propre piqûre ${ }^{66}$.

62 Traduction de P. Savinel, in Arrien, Histoire d'Alexandre. L'Anabase d'Alexandre le Grand, suivi de Flavius Arrien entre deux mondes, par P. VIDAL-NAQUE', Paris, 1984. Le texte grec est celui de la Loeb Classical Library (I, 1976 [1929]). Lire, sur ce passage, les commentaires de Marsden, o.c. (n. 53), p. 97-98, 165-166.

63 Sur le riche champ de représentation que couvre ce mot, voir $D E L G$, $\mu \eta \chi \alpha v \eta ́$, ainsi que les pages VII et VIII de l'introduction du Livre X du De Architectura de Vitruve, par CAltebat - FleurY, o.c. (n. 53), pour le sens qui nous intéresse ici.

64 C'est ce que comprend Savinel., o.c. (n. 62), p. 13. Un passage des Histoires de QuinteCurce confirme et précise l'identification de ces machines qui couvrent les Grecs pendant la traversée du fleuve (VII, 9, 7-8) : les engins qui accablent les Scythes sont des catapultes 'à torsion' (tormenta) lançant des 'traits' (tela), probablement des scorpions.

65 L'archer scythe est une sorte d'bipposkorpios, si on me permet de forger ce mot composé.

66 Il n'y a pas là qu'une image. Avant la naissance de l'esprit scientifique et le triomphe de la rationalité, la pensée médicale ancienne reconnaissait l'efficacité d'un principe que l'on pourrait 
«Bête à flèches » pour les Grecs, le scorpion incarne le côté noir de l'arc et de la flèche. Dans la fable 293 d'Esope, comme le dit explicitement la morale, il tient de façon significative le rôle du méchant ${ }^{67}$. Maléfique et mortifère, tel apparaît le scorpion. Il est aussi le doublet animal du « sous-guerrier » à l'arc, tant décrié sur les champs de bataille, d'Homère à Euripide. On peut ainsi mettre en parallèle le scorpion qui sort de sa cachette pour chasser, prêt à décocher son aiguillon, et Pâris, l'archer lâche et efféminé de l'Iliade. L'affrontement avec Diomède est, à ce propos, particulièrement significatif (XI, 369-395). Pâris choisit le moment où Diomède dépouille de ses armes le guerrier qu'il vient de tuer et où il n'est donc pas sur ses gardes, pour lui décocher une flèche à la dérobée (v. 373-375). C'est caché et bien à l'abri de son adversaire, qu'il tire sur lui : l'archer est accoté à une stèle ( $\sigma \tau \eta^{\prime} \lambda \eta$

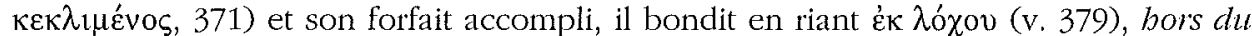
lieu oú il est embusqué, ce que Paul Mazon rend bien par hors de sa cacbette ${ }^{68}$. Pâris touche Diomède au pied (v. 377-378). Le coup n'est guère anodin : il se place dans la logique de la condamnation morale de l'arc et de l'archer en Grèce ancienne. Mais le pied, c'est aussi la partie du corps humain réputée la plus exposée à la piqûre du scorpion. Ainsi, Pâris se tient en embuscade et frappe avec son arc tout comme le fait le scorpion quand il sort de sa cachette.

Considérons pour finir un des arguments que le défenseur de l'archer oppose au porte-parole de l'hoplite, dans la fameuse controverse de l'Héraclès d'Euripide (v. 198-199): il repousse les ennemis qui voient des traits aveugles ( $\tau v \varphi \lambda \lambda_{0} \varsigma$ $\tau o \xi \varepsilon v ́(\alpha \sigma r)$ les atteindre sûrement ${ }^{69}$. Ces traits aveugles, vus par ceux qui les reçoivent sont en fait des flèches qu'on ne voit pas venir. Pareillement, les "flèches " du scorpion « décochées » à l'improviste, sont aussi « aveugles », c'est-à-dire invisibles et imprévisibles, que celles de Pâris placé en embuscade ou celles de l'archer d'Euripide. D'autant plus « aveugles », notons-le, que le scorpion est un animal crépusculaire et nocturne, qui sort la nuit pour chasser.

Que conclure au terme de cette étude articulée autour du scorpion?

J'ai montré combien le scorpion est à sa place dans l'entourage d'Apollon à Claros. Iobolos, «lanceur de flèches et de venin » et bathyplex, « qui pique profondément », le scorpion est un guerrier à l'arc, un Scythe habile à décocher des flèches venimeuses, ou bien une vivante catapulte à flèches. Tel apparaît l'« animal-archer » qu'Apollon repousse de son sanctuaire. Ce scorpion Toxophoros, qui porte l'arc comme Apollon, mérite tout autant que son chimérique parent, le martichore, la très apollinienne appellation de zôon hékebolon, d'animal qui lance ses flèches au loin.

formuler ainsi : « celui qui est la cause de la maladie, ou qui a fait la blessure, est le mieux à même de la guérir. " Cette attitude 'sympathique' fait partie d'un fonds commun à toutes les médecines traditionnelles, ce qu'illustre pleinement la pratique médicale de l'Antiquité. Contre les piqûres de scorpion, par ex., la pharmacopée des Grecs et des Romains faisait une bonne place au scorpion lui-même, pris comme topique ou en fumigation, mangé cuit ou bien bu dans du vin. Voir par ex. le De Medicina de Celse (V, 27, 5A) et l'Histoire Naturelle de Pline (XI, 90 et XXIX, 91). Ces pratiques thérapeutiques relèvent de la pensée 'sauvage' ou 'magique' étudiée par Cl. Lévi Strauss. Voir l'exemple qu'il donne, o.c. (n. 17), p. 15, de l'attouchement avec un bec de pic utilisé contre les maux de dents par les Iakoutes de Sibérie.

67 En jouant à attraper des sauterelles, un enfant échappe de peu à l'aiguillon venimeux d'un scorpion.

68 Homère, lliade, t. II, Paris, Les Belles Lettres, réimpr, 1972 [1937].

69 Trad. de L. Parmentier, Euripide, t. III, Paris, Les Belles Lettres, réimpr. 1994 [1923]. 
Mais embusqué, nocturne, décochant un trait imprévisible et empoisonné, le scorpion semble bien l'archer "noir » d'Apollon, chargé des valeurs négatives de la flèche : la meurtrière qui avance au ras du sol, qui frappe par surprise, empoisonne et fait souffrir ${ }^{70}$. "Signe de chute », le scorpion apparaît alors comme le doublet animal de l' « arc-moins » ou de la «flèche-moins $» 71$.

À Claros, c'est l'archer Apollon - qui est aussi médecin - qui apparaît comme le meilleur antidote contre cet archer venimeux qu'est le scorpion. Sur le seuil de son sanctuaire, il est le Prostaterios, celui qui se tient devant, celui qui protège du mal "toxique » qu'est le scorpion. Montant la garde avec son arc, il est l'Alexikakos et l'Apotropaios, celui qui écarte, qui détourne les maux ${ }^{72}$, l'Hélacrergos, celui qui repousse au loin $^{73}$ avec ses flèches. L'Archer divin de Claros tient à distance à coup de flèches un « animal à flèches ».

Apollon n'est pas seul, pour chasser de son sanctuaire de Claros des animaux venimeux, qui piquent ou qui mordent. Les quatre vers de Nicandre indiquent clairement la tâche apotropaïque que le dieu a assignée au frêne. Chargé de signes et d'images concrètes, cet arbre apparaît comme un gardien très qualifié contre des animaux-archers décochant des flèches empoisonnées. De façon plus générale, il trouve parfaitement sa place aux côtés d'un Apollon clarien, oraculaire, archer et médecin.

Il n'y a plus aujourd'hui de frênes à Claros, mais le discours de Théophraste sur. cet arbre dans les Recherches sur les plantes et celui des botanistes modernes ${ }^{74}$ permettent d'identifier, avec une certaine assurance, l'espèce qui peuplait le sanctuaire. On peut écarter d'emblée le frêne commun (Fraxinus excelsior L.) qui « couvre pratiquement toute l'Europe sauf les contrées méridionales » ${ }^{75}$ et qui est remplacé en Grèce par une espèce très voisine, le frêne oxyphylle ou à feuilles aiguës

70 On peut voir dans le scorpion le pendant symbolique du dauphin, véritable 'flèche vivante' d'Apollon qui porte les valeurs positives de la flèche en vol, celle qui fuse dans la lumière, aérienne et salvatrice.

71 Voir J. Le Goff et P. Vidal-Naquet, "Lévi-Strauss en Brocéliande. Esquisse pour une analyse d'un roman courtois", in R. Bellour et C. Chément (éds), Claude Lévi-Strahiss, textes de et sur Cl. Lévi-Strauss, Paris, 1979, p. 265-319. Les auteurs ont bien montré l'ambiguité fondamentale de l'archer et de son arme : médiéval ou grec, « l'arc est un signe dont la valeur n'est donnée que par la position qu'il occupe dans le système » (p. 275). En fonction du contexte dans lequel il est utilisé et du statut de celui qui le porte, il pourra être 'signe de chute' ou 'signe de remontée' (p. 277), au point que P. Vidal-Naquet a pu parler d'un 'arc-moins' et d'un 'arc-plus' (Le chasseur noir. Formes de pensée et formes de société dans le monde grec, Paris, 1983 [1981], n. 48, p. 193).

72 Sur la fonction protectrice et conjuratoire que recouvrent ces trois épiclèses d'Apollon, je renvoie à M. Detienne, Apollon le couleau à la main. Une approcbe expérimentale du polythéisme grec, Paris, Gallimard, 1998 (Bibliotbèque des Sciences bumaines), p. 96, 123-125.

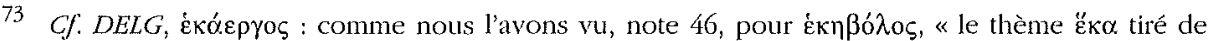

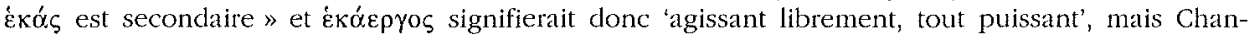

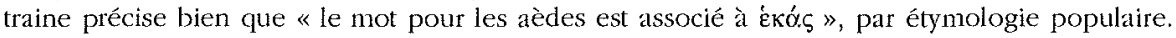

74 Outre l'édition, la traduction et les très riches commentaires de S. Amigues, Théophraste, Recberches sur les plantes, t. II et III, Paris, Les Belles Lettres, 1989 et 1993, nous avons, pour les informations d'ordre botanique, utilisé trois ouvrages: H. Duflot, Le frêne en liberté, Paris, Institut pour le Développement forestier, 1995; G. Motel, Le frêne, Actes Sud, 1996 (Le nom de l'arbre) et B. Bertrand, Le Frêne, arbre des centenaires, 1997 (Le compagnon végétal), vol. 4.

75 Motel, o.c. (n. 74), p. 19. 
$(F \text {, oxyphylla Bieb. ou } F \text {, angustifolia Vahl })^{76}$. Un second « candidat » possible est le frêne orne ou frêne à fleurs ( $F$. ornus L.) : très commun autour de la Méditerranée, il se rencontre depuis l'Espagne jusqu'en Turquie et notamment « partout en Grèce à des altitudes très variables ${ }^{77}$. Ce sont les deux espèces de frênes que connaît la flore grecque et dont on peut penser qu'elles poussaient sur la côte ionienne. Mais de ces deux espèces, quelle est celle du bois sacré de Claros? L'étude comparée de leur écologie et de leur morphologie plaide en faveur du frêne oxyphylle.

Le premier argument tient à la taille de ce frêne. Théophraste distingue nettement les deux espèces ( $R P$ III, 11, 3):

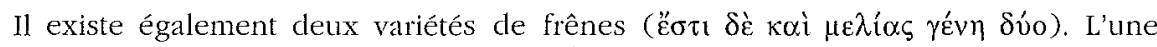

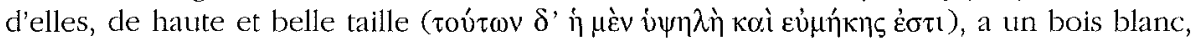
à fibres droites, plus tendre, plus dépourvu de nœuds et de madrures; l'autre, plus

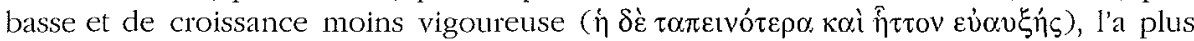
raboteux, plus dur et plus jaune.

Et plus loin (III, 11, 4) :

L'espèce à bois lisse habite surtout les vallées profondes et les lieux humides ( $\pi \varepsilon p \grave{i}$

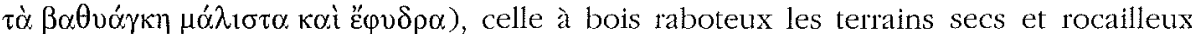

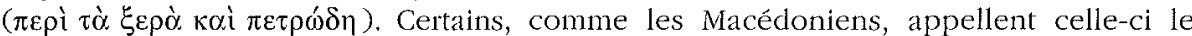

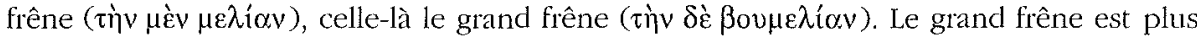

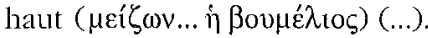

Dans le "grand-frêne » (boumelia ou boumelios) ${ }^{78}$, remarquable par sa baute et belle taille, on reconnaît le frêne oxyphylle, qui peut atteindre 25 mètres de haut, alors que le « frêne »-melia, espèce plus basse et de croissance moins vigoureuse, comme dit Théophraste, correspond au frêne orne, "petit arbre et souvent arbrisseau de $7-8 \mathrm{~m} .{ }^{79}$. La taille très élancée du frêne oxyphylle s'accorde mieux que celle du frêne one avec le texte de Nicandre: Apollon a caché le vallon sous les frênes $\left(\mu \varepsilon \lambda i n \sigma_{1} \kappa \alpha \lambda \hat{u} \psi \alpha \varsigma\right)$. Par là même, c'est aussi lui qui répond le mieux à l'image récurrente de l'alsos ombragé et frais. Les deux autres arguments tiennent à l'écologie et à la morphologie du frêne oxyphylle. Ils vont guider notre enquête et lui ouvrir de vastes horizons apolliniens.

Tout d'abord, l'écologie du frêne oxyphylle s'accorde particulièrement avec le site du sanctuaire clarien, ainsi qu'avec la mantique par absorption d'eau qui y est pratiquée. L'examen des sols sur lesquels prospèrent les deux espèces de frênes vérifie le texte de Théophraste. Le boumelia préfère les vallées profondes ( $\tau \dot{\alpha} \beta \alpha \theta v \dot{\alpha} \eta$ ) et les lieux bumides ( $\varepsilon_{\varphi} \varphi \delta \rho \alpha$ ), or le frêne oxyphylle aime se développer sur les sols humides ${ }^{80}$ : il est « beaucoup plus hygrophile ${ }^{81}$ que le frêne orne. Ce dernier, le

76 Voir Amigues, o.c. (n. 74), n. 10, p. 160 à RP III, 11, 3 et Duflot, o.c. (n. 74), p. 20.

77 Amigues, o.c. (n. 74), n. 10, p. 160 à RP III, 11, 3. Voir aussi Bertrand, o.c. (n. 74), p. 18 et Motel, o.c. (n. 74), p. 26.

78 Cf. DELG, $\beta$ ov-: Chantraine relève l'emploi de ce préfixe augmentatif dans les noms de plantes et cite l'exemple du frêne.

79 Amigues, o.c. (n. 74), n. 10, p. 160 à RP III, 11, 3. Bertrand, o.c. (n. 74), p. 18, indique qu'« il dépasse rarement $10 \mathrm{~m}$. de haut ».

80 Cf. Bertrand, o.c. (n. 74), p. 17.

81 Amigues, o.c. (n. 74), n. 10, p. 160 à $R P I I I, 11$. 
melia des Grecs, aime au contraire les terrains secs ( $\xi \varepsilon \rho \alpha \dot{)}$ et rocailleux ( $\pi \varepsilon \tau \rho \omega ́ \delta \eta$ ) et c'est dans les mêmes termes que G. Motel qualifie le milieu de prédilection du frêne orne : «il demande (...) des sols secs et pierreux ${ }^{82}$. Le frêne oxyphylle est, comme le frêne commun, un arbre véritablement « assoiffé »: H. Duflot note que « chaque arbre élève ainsi, en une journée d'été, plus de deux cent litres d'eau " ${ }^{83}$. Son existence est étroitement tributaire des eaux souterraines ou de surface. Il étanche sa soif en peuplant les sols humides et frais, les fonds de vallée notamment ${ }^{84}$. Ce tableau s'accorde très exactement avec le site de Claros décrit par Nicandre et Pausanias : la frênaie sacrée s'étend au fond d'un profond vallon ( $\alpha$ ủ $\omega^{\prime} v \alpha \beta \alpha \theta \dot{v}$ ) ${ }^{85}$ tapissé de terre

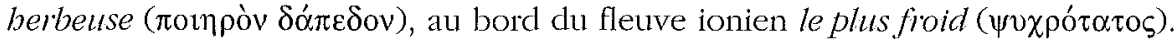

L. Robert a dit l'adéquation entre le site du sanctuaire tel qu'on peut l'apprécier aujourd'hui et le batbys aulôn de Claros dont parle le prêtre d'Apollon : «c'est une expression très juste pour désigner cette vallée à la fois étroite et longue; que l'on vienne du village d'Ahmetbeyli, au nord de Claros, ou de la mer, ou qu'on regarde Claros depuis les hauteurs, on est frappé par la vérité des mots de Nicandre ${ }^{86}$. Ce profond vallon correspond aux goûts du frêne oxyphylle et il satisfait aussi les goûts d'Apollon. Le batbys aulôn de Claros est un excellent exemple de ce que je propose d'appeler un «site en adyton». Les Grecs, en effet, semblent avoir recherché pour les sanctuaires oraculaires d'Apollon ce genre de site en creux, secrètement enfoui ou retiré, qui forme comme un adyton naturel, à l'image de la clépression de Didymes et des deirades de Delphes et d'Argos ${ }^{87}$.

Le vallon de Claros fournissait aussi au frêne oxyphylle toute l'eau qui lui était nécessaire ${ }^{88}$. Cette eau affleurait sous le temple où elle jouait un rôle essentiel dans le

O.c. (n. 74), p. 28.

83 O.c. (n. 74), p. 84. Cf. p. 85 : l'auteur décrit l'imposant système racinaire très efficace pour' pomper l'eau.

84 Voir Bertrand, o.c. (n. 74́), p. 13, 21, 37 et Duflot, o.c. (n. 74), p. 86.

85 On remarque que c'est le même terme qui désigne, chez Théophraste, le domaine du 'grand frêne'-boumelia et chez Nicandre, le site de Claros : $\beta \alpha \theta v \alpha \gamma \kappa n ́ s$, aux vallées profondes est un des

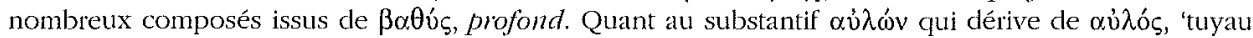

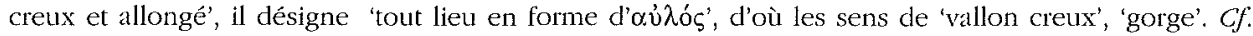
$D E L G, s . v$.

86 O.c. (n. 3), p. 528. Voir aussi, L. Rober't, "L'oracle de Claros", in Ch. Delvore, G. Roux (éds), La civilisalion grecque de l'antiquité à nos jours, Bruxelles, 1967, p. 307.

87 Voir H.W. Parke, The oracles of Apollo in Asia Minor, Londres, 1985, p. 1-2 et J. Fontenrose, Didyma. Apollo's Oracle, Cult, and Companions, Berkeley, University of California Press, 1988, p. 1: les auteurs ont bien reconnu, comme peut le faire le visiteur, la cuvette (bollow) dans laquelle se trouve le Didymeion d'Apollon. A Delphes, le dieu a construit son temple dans une deiras (cf. l'Hymne ps.-bom. à Apollon, 281-282), haute terrasse adossée au versant méridional du Parnasse. A Argos, le sanctuaire de la Deiras (cf. Paus., II, 24, 1-2) est niché sur le flanc sud-ouest de la colline du prophète Élie, dans le vallonnement entre cette colline et la colline de la Larissa. Les recherches menées dans le cadre de ma thèse me conduisent à me demander s'il n'existerait pas un rapport entre les adyta d'Apollon - l'adyton naturel que forme le site et l'adyton construit au fond des temples oraculaires - et la nature profonde de la mantique d'un dieu archer et musicien qui oblige précisément à 'descendre', à 'entrer' au plus profond de soi, dans son 'adyton intérieur'.

88 Les frênes célébrés par Nicandre n'ombragent plus aujourd'hui le domaine d'Apollon, mais l'eau qui les désaltérait est toujours là et même trop au goût des archéologues, la nappe phréatique ayant considérablement monté depuis l'Antiquité. "Notre ennemie, c'est l'eau », déclarait RoBert, l.c. (n. 3), p. 529, en 1953. En effet, plus que les eaux de surface, la nappe souterraine, très proche, a 
déroulement de la séance oraculaire, ainsi qu'en témoignent Tacite et Jamblique notamment. Dans un passage des Annales, l'historien romain raconte la consultation de l'oracle par Germanicus, en 18 de notre ère : un prêtre (sacerdos) descend dans une grotte (in specum degressus) et puise de l'eau à une source mystérieuse (bausta fontis arcani aqua) avant de répondre aux questions posées ${ }^{89}$. Presque trois siècles plus tard, dans Les mystères d'Égypte, le néoplatonicien Jamblique examine le fonctionnement des trois grands oracles apolliniens de Claros, Delphes et Didymes. Il commence par l'oracle clarien auquel il consacre un long développement et marque très fortement le rôle de l'eau dans la consultation (III, 11 - 124, 10 sq.):

D'abord l'oracle de Colophon. Tous conviennent qu'il prophétise par le moyen de

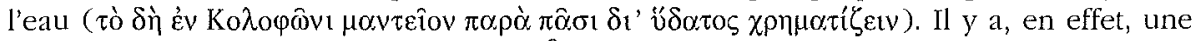

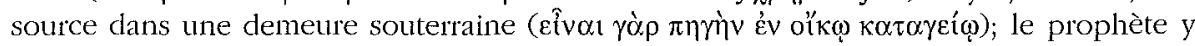

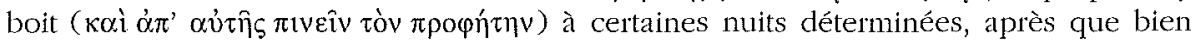

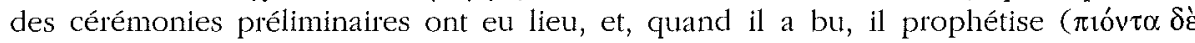
$\chi \rho \eta \sigma \mu \varphi \delta \varepsilon i ̂ v)$ sans plus être visible aux spectateurs présents. Que cette eau-là soit divinatrice, c'est ce qui va de soi ( $\left.\pi \rho{ }^{\delta} \delta \eta \lambda \mathrm{ov}\right)^{90}$.

Il s'agit donc d'un oracle par absorption d'eau, comme l'a confirmé le dégagement complet des deux salles souterraines de l'adyton: au fond du temple, dans la seconde pièce près de la poterne qui fait communiquer les deux salles, L. Robert a retrouvé « un puits rectangulaite de $1,41 \mathrm{~m}$ sur $96 \mathrm{~cm}$ (...) réservé dans le dallage » et il indique que « primitivement», l'eau était « au fond de la salle (...), derrière une sorte de balustrade continue $»^{91}$. Là était la source sacrée à laquelle puisait le prophète $^{92}$. Tout comme le prophète qui descendait au plus profond du temple, dans la partie la plus reculée de l'adyton, pour boire l'eau inspiratrice, les frênes puisaient eux aussi l'eau d'Apollon, au fond de ce vallon-adyton, dans la nappe souterraine proche qui alimentait la source sacrée. Les Grecs avaient reconnu, comme en témoigne Théophraste, le goût du « grand frêne »-boumelia pour les vallées profondes ( $\beta \alpha \theta u \alpha ́ \gamma \kappa \eta)$ et les lieux bumides ( $\ddot{\varepsilon} \varphi v \delta \rho \alpha$ ). On peut supposer alors que, dans le contexte clarien, chez le maître de la mantique, les frênes buveurs d'eau de l'alsos leur soient aussi apparus comme des arbres en quelque sorte inspirés par Apollon, à l'instar du prophète.

beaucoup gêné le déblaiement des monuments. Ainsi les tunnels qui menaient à l'adyton ont été dégagés à l'aide d'une pompe et les bottes aux pieds. Voir aussi J. DE LA Genière, "Le sanctuaire d'Apollon à Claros. Découvertes récentes", CRAI (1992), fasc. I, p. 197, 208 et l.c. (n. 6), p.16-17.

89 TACrre, Annales II, 54, texte établi et traduit par P. Wuilleumier, Les Belles Lettres, t. I, 1974.

90 Texte établi et traduit par E. des Places, Paris, Les Belles Lettres, 1996 [1966]. Sur ces deux textes de Tacite et Jamblique, on peut se reporter à PARKe, o.c. (n. 87), p. 136-138, 219-224, ainsi qu'à J. \& L. Robert, "Décret de Colophon pour un chresmologue de Smyrne appelé à diriger l'oracle de Colophon", BCH 116 (1992), p. 286-288.

91 O.c. (n. 86), p. 311. Seul le prêtre, probablement, entrait dans cette salle de l'oracle - la 'caverne' de Tacite, la 'demeure souterraine' de Jamblique -, l'adyton proprement dit, pour y recevoir l'inspiration en buvant l'eau du dieu. Il faut imaginer le personnel de l'oracle et les consultants assis dans la première salle, attendant et écoutant la réponse d'Apollon, avant d'emprunter à nouveau les longs, étroits et sinueux tunnels qui les ramèneraient dans le pronaos, à la surface.

92 Sur le déroulement de la consultation, voir Robert, l.c. (n. 86), p. 309-312; PARKe, o.c. (n. 87), p. 138-139, 220-224, ainsi que R. LANe Fox, Païens et cbrétiens, Toulouse, Presses Universitaires du Mirail, 1997 [1986] (Amphi 7-Histoire), p. 183-187. 


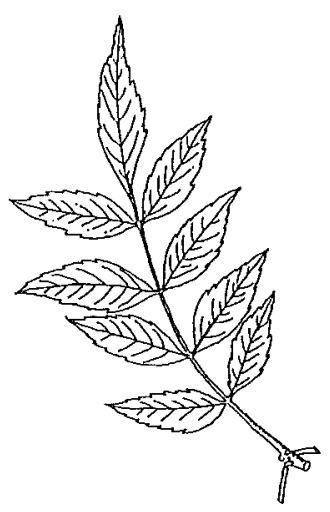

Doc. 6

La feuille composêe du frêne oxyphyle

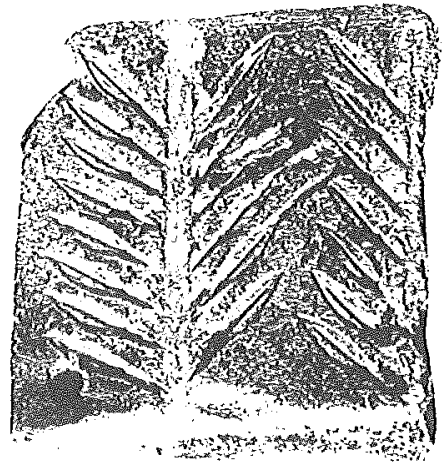

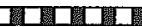

Doc. 7

Moule pour pointes de flèches

Venons-en maintenant au troisième et dernier argument en faveur du frêne oxyphylle. Il tient au caractère même qui a donné son nom à cette espèce : ses feuilles pointues. Théophraste l'avait déjà noté. Il indique à propos des deux espèces de frênes (RP III, 11, 3) :

Leurs limbes foliaires rappellent pour la forme une feuille de laurier ( $\tau \dot{\alpha}, \delta \grave{\varepsilon} \varphi v ́ \lambda \lambda \alpha$

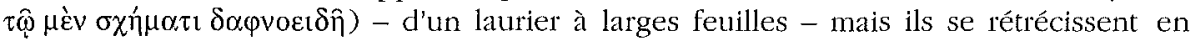

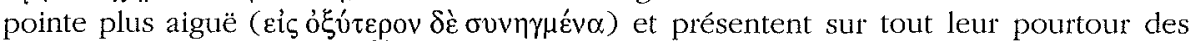
sortes de dents mucronées ${ }^{93}$. (...) Ces folioles sont disposées individuellement le long d'une sorte de nervure unique, par paires correspondant aux nœuds, les paires étant

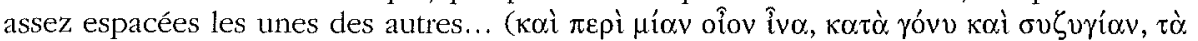

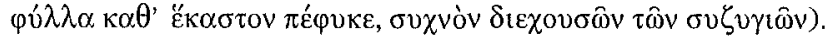

Comme le note S. Amigues, Théophraste décrit ici le principe de la «feuille composée » du frêne oxyphylle ${ }^{94}$, avec ses folioles opposées par paires le long du rachis central (document 6). Or, la forme même de la feuille composée évoque la technique utilisée pour couler au moule les pointes de flèches en bronze. C'est ce que montre le fragment d'un tel moule découvert en 1975 dans une ville de la fin de l'Age du Bronze, sur la côte sud-est de Chypre, par la Mission suédoise que dirige Paul Åström ${ }^{95}$. Il s'agit d'une plaque carrée en pierre de chaux, de $23 \mathrm{~cm}$ sur $24,5 \mathrm{~cm}$, épaisse de $6,2 \mathrm{~cm}$, brisée d'un côté et percée de deux trous, qui permettaient d'ajuster l'autre moitié du moule au moyen de nouds. Les pointes sont gravées en creux sur un côté de la plaque (document 7) : du canal de coulée central partent, d'un côté,

93 Peu après, Théophraste précise à propos de la feuille du 'grand frêne' que ses folioles ( $\varphi \hat{u} \lambda \lambda \alpha)$ sont plus longues ( $\mu \alpha \kappa \rho o ́ \tau \varepsilon \rho \alpha$ ), plus étroites ( $\sigma \tau \varepsilon v o ́ \tau \varepsilon \rho \alpha$ ) et d'un vert de poireau. Les folioles du frêne oxyphylle sont effectivement « étroites, effilées et bordées de dents aiguës » (Amigues, o.c. [n. 74], n. 15, p. 161 à III, 11, 3), alors que celles des frênes commun et orne sont plus arrondies (Duflot, o.c. [n. 74], p. 21 et Motrel, o.c. [n. 74], p. 21, 24-27).

94 O.c. (n. 74), n. 12, p. 160-161 à III, 11, 3.

95 Le site, au bord du lac salé de Larnaca, est fouillé, depuis 1971, par l'Université de Göteborg. Voir V. Karageorghis, Les anciens Chypriotes. Entre Orient et Occident, Paris, 1991, p. 69, 74, 101, et P. Åsтröм, "Les fouilles de Hala Sultan Tekké", Dossiers d'Arcbéologie, 205 H (juillet-août 1995), p. $42-47$. 
huit pointes de flèches et de l'autre six. Le long du côté brisé court un autre canal dont partent sept pointes ${ }^{96}$. L'ensemble rappelle étrangement l'empreinte d'une feuille composée fossilisée dans la pierre : les pointes se font face, par paires, le long d'un canal de coulée auquel elles sont attachées par leur soie, comme des folioles disposées, par paires, le long du rachis auquel elles tiennent par leur pédoncule ${ }^{97}$. Les têtes de flèches elles-mêmes sont très semblables à des folioles de feuilles composées, comme celles du frêne oxyphylle : elles sont étroites, effilées, longues de $7 \mathrm{~cm}$ et creusées d'une profonde rainure médiane qui leur donne une véritable nervure ${ }^{98}$. Une pointe de flèche trouvée sur le site fait fortement penser, elle aussi, à une foliole (document $8 \mathrm{a})^{99}$. G. Hult la rapproche d'une pointe inventoriée par Hans-Günter Buchholz dans sa typologie des pointes de flèches égéennes de l'Age du Bronze (document $8 \mathrm{~b})^{100}$. Elle appartient à un type de pointes d'origine orientale, répandu en Syrie et à Chypre, connu en Anatolie, à Troie, à Rhodes et attesté dans le monde égéen à partir de ca 1450 avant J.-C. H,-G. Buchholz note à propos de ce type de pointe que « la forme lancéolée de la feuille est relativement fréquente ${ }^{101}$ : elles sont en effet foliacées d'aspect, dotées d'une large nervure médiane et d'un long pédoncule (document $8 \mathrm{c})^{102}$. Aujourd'hui, en terme d'archerie, ce type de pointe peut se dire « en feuille de laurier» et c'est bien de la foliole du laurier - arbre apollinien s'il en est - que Théophraste ( $R P$ III, 11,3), nous l'avons vu, rapprochait la foliole du frêne $^{103}$. Ainsi, à Claros, les frênes dont l'Archer a recouvert son sanctuaire pour tenir

96 Ce fragment de moule est exposé au Musée de Chypre à Nicosie (vitrine consacrée au travail du métal, salle XII) avec une réplique de la pièce qu'il a servi à mouler : une coulée centrale et ses quatorze flèches attenantes. Voir le rapport de fouilles de G. Hult, "Area 8. The 1975 Campaign", Hala Sultan Tekke 4, SIMA 45, 4 (1978), p. 16-94. Le Musée de Delphes abrite quatre pointes de flèches en bronze des IV ${ }^{\mathrm{e}}$ Ou III ${ }^{\mathrm{e}}$ siècles av. J.-C., partiellement réunies, au niveau de la pointe, par un canal de coulée, ce qui les apparente fortement au moule chypriote. Voir Cl. Rollex, "Les Bronzes", Guide de Delphes. Le Musée, Paris, 1991 (EFA, Sites et monuments, 6), fig. 1, p. 143.

97 A.J. Reinach, auteur de l'article "Sagitta" dans le Dictionnaire des Antiquités grecques et romaines IV, p. 997-1000) ne parle que du très botanique 'pédoncule' pour qualifier la soie, c'est-àdire la partie mince et allongée à la base de la pointe, qui s'enfonce dans la hampe de la flèche.

98 On peut faire une description très végêtale de ce moule à pointes de flèches et il est remarquable que Hulr, l.c. (n. 96), p. 83, dans son commentaire archéologique, ait été entraînée vers une description de ce type : " one channel branching out to eight arrow-heads on one side and six on the other », c'est-à-dire que le canal de coulée étend ses branches, se ramifie sur les côtés en poussant des pointes de flèches. L'auteur parle de la nervure (rib) qui traverse chaque 'arrow leaf', chaque pointe de flèche à l'apparence de feuille. L'expression 'tree casting' utilisée pour désigner un système de coulée en forme d'arbre est aussi très significative.

99 Cf. l.c. (n. 96), p. 81, nº N1114 : très semblable à celles qui ont pu être coulées dans le moule, elle est 'leaf shaped', en forme de feurlle, pourvue d'une soie et montre, sur un côté, les traces d'une nervure médiane. Voir aussi p. 83-85; fig. 149-150, p. 90.

${ }^{100}$ H.-G. Buchiolz, "Der Pfeilglätter aus dem VI. Schachtgrab von Mykene und die helladischen Pfeilspitzen", JDAI 77 (1962), fig. 7, p.11 et commentaires p. 26-27.

101 L.c. (n. 100), p. 26 : « relativ haüfig ist die Lanzettform des Blattes, » On relève ici aussi le vocabulaire très végétal utilisé pour désigner une tête de flèche qui évoque une fouille (Blatt) en forme de lance, 'lancéolée' comme disent les botanistes.

102 Cf. l.c. (n. 100), p. 22, les photographies des 14 pointes du type VIIa, fig. 13a à 13 n.

103 Cette forme 'en feuille de laurier' est attestée parmi les nombreux types de pointes de flèches connus en pays grec, mais les sources semblent ignorer une appellation de ce genre, inspirée par la botanique. Notons que certains modèles traditionnels japonais de pointes en acier portent 
à l'écart des « animaux-archers », brandissent dans leurs frondaisons des feuilles qui évoquent étrangement des pointes de flèches au sortir du moule. Le frêne oxyphylle, avec son feuillage sagitté, convient donc tout particulièrement pour abriter le sanctuaire clarien du dieu à l'arc et le protéger contre les agressions d'animaux prompts à décocher leurs flèches.

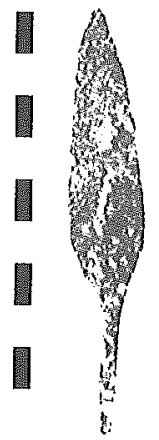

Doc. $8 \mathbf{a}$

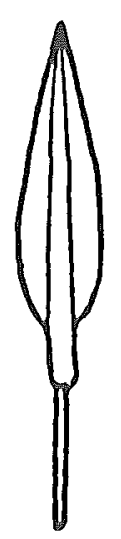

Doc, 8 b

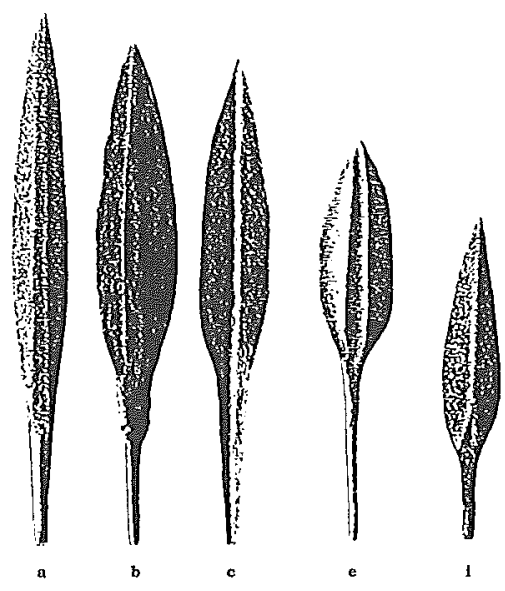

Doc. $8 \mathrm{c}$

Des pointes de flèches en forme de folioles

Mais ce n'est pas tout. On peut étendre ce rapprochement entre les feuilles des frênes d'Apollon à Claros et les pointes de flèches: le bois du frêne a très bien pu intéresser, sur les bords de l'Égée, les facteurs d'arcs et les fabricants de flèches.

Rappelons tout d'abord que notre documentation, à de notables exceptions près, est particulièrement avare sur les essences utilisées pour équiper l'archer dans le monde grec : les Recherches sur les plantes de Théophraste, par exemple, dont on pouvait attendre des renseignements explicites, sont muettes sur le sujet, si ce n'est

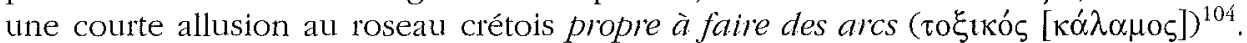
Or, il suffit d'interroger les archers ailleurs dans le temps et dans l'espace, pour constater que, quand l'environnement végétal s'y prête, le frêne est toujour's en bonne place dans leur arboretum. La «toxophilie » de son bois est reconnue depuis longtemps : les bras en frêne, élastiques et résistants ${ }^{105}$, supportent les forces d'étirement et de compression qui s'exercent sur la face externe et la face interne de l'arc quand il est bandé et armé. C'est ainsi qu'à côté d'arcs en bois d'orme, les tourbières du Mésolithique danois ont livré des arcs simples - faits d'une seule pièce de bois - en frêne, comme ceux trouvés à Brabrand et datés de 3500 avant notre ère ${ }^{106}$. Pendant

les noms évocateurs de 'feuille de bambou', 'feuille de saule' ou 'feuille de camélia'. Voir R. Roтr, Historre de l'Arcberie. Arc et arbalète, Montpellier, 1992, p. 117.

104 RP IV, 11, 11. Voir les commentaires ad loc. de Amgues, o.c. (n. 74).

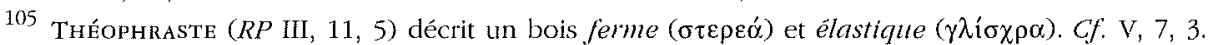
D. Coutro't, Le bois et ses industries, Paris, 1997 (Que sais-je ?), p. 29, souligne que « c'est un excellent bois pour les manches d'outils, pour les éléments cintrés d'articles de sport : luges, javelots, arcs, etc. ». Voir aussi Amigues, o.c. (n. 74), n. 10, p. 160 à III, 11, 3 et BerTrANd, o.c. (n. 74), p. 81-95.

106 Voir M. Orlac, "Mésolithique", Dictionnaire de la Prébistoire, Encyclopaedia Universalis, Paris, Albin Michel, 1999, p. 665. 
tout le Moyen Âge, en France, les arcs de frêne côtoient ceux d'érable, d'orme ou d'if $^{107}$. Séduit par le tir à l'arc au Brabant, lors de la campagne de 1814 contre Napoléon, Johann Peter Eckermann, confident et disciple admiratif de Goethe, s'est aussi tourné vers le frêne pour fabriquer ses premiers arcs, autour de Weimar ${ }^{108}$. Enfin, les facteurs d'arcs américains, grands connaisseurs de l'archerie nord-amérindienne, relèvent souvent à quel point celle-ci appréciait les qualités mécaniques du bois de frêne ${ }^{109}$. Si le frêne donne de bons arcs, il donne aussi de bonnes flèches, comme en témoigne, par exemple, l'archéologie scythe ${ }^{110}$.

On peut dès lors s'autoriser l'hypothèse suivante : intimement familiarisés, comme la plupart de leurs contemporains, avec la flore qui les environnait, connaissant parfaitement les propriétés des nombreux bois qu'ils travaillaient et qu'ils utilisaient, les facteurs d'arc en pays grec - comme les fabricants de flèche -, n'ont probablement pas manqué d'apprécier les qualités qui font du frêne un très bon bois d'arc, mais aussi un excellent bois de flèches ${ }^{111}$.

Si elle n'a pas retenu l'utilisation du frêne comme bois d'arc et de flèches, du moins de façon explicite, la tradition grecque et plus particulièrement homérique, en connaît une autre qui fait aussi du bois de frêne un bois qui pique et qui transperce : le frêne, c'est le bois de la lance et $\mu \varepsilon \lambda$ ín signifie aussi chez Homère javeline, pique au manche de frêne ${ }^{112}$. Dans l'tliade, cette lance de frêne arme de nombreux héros, tant grecs que troyens : Ajax, Enée, Hector et surtout Achille ${ }^{113}$. Le frêne est, avec le cornouiller, le bois qui convient le mieux pour la lance de l'hoplite et la lance ou

107 Voir Roth, o.c. (n. 103), p. 71 et J. Daimé, "L'arc en France au Bas Moyen Age : fabrication et techniques", in M.-Cl. Amouretti, G. Comet (éds), Artisanat et matériaux. La place des matériaux dans l'bistoire des techniques, Université de Provence, 1998 (Cabiers d'bistoire des techniques, 4), p. $62-63$.

108 Lire la conversation du $1^{\mathrm{er}}$ mai 1825 in Conversations de Goethe avec Eckermann, trad. de J. Chuzeville, nouv. éd. revue et présentée par Cl. Roëss, Paris, Gallimard, 1988 [1949], p. 480-491.

109 Voir R. et G. LAUBIn, American Indian Archery, Norman/Londres, University of Oklahoma Press, réimpr. 1990 [1980] (The Civilization of the American Indian series, 154), p. 22, 59, 73, 87, 89, 120; D. Wallentine, Making Indian Bows and Arrows... The Old Way, Liberty, Utah, Eagle's View Publisbing Edition, 1988, p. 3-4 et J. Hamm, L'arc indien. Comment fabriquer vous-même les arcs, flèches et carquois des Indiens d'Amérique du Nord, 1994 [1989], p. 19-20.

110 Voir V. Schiltz, Les Scytbes et les nomades des steppes. VII ${ }^{e}$ siècle avant J.-C. - Fr siècle après J.-C., Paris, Gallimard, 1994 (L'Univers des Formes), p. 389 et Lebedynsky, o.c. (n. 61), p. 156. Le frêne est aussi préconisé pour les flèches de guerre dans le Toxophilus, traité d'archerie anglais rédigé par R. Ascham en 1544. Voir Daimé, l.c. (n, 107), p. 63-64. De la même façon, la chasse à l'arc moderne fait encore grand cas du frêne pour confectionner de bons fûts de flèches. Voir Ph. Gras et L. Barbier, Manuel de la cbasse à l'arc, Paris, Éditions du Gerfaut, Office National de la Chasse, 1996, p. 14. Ces témoignages sont d'autant plus précieux, qu'ils concernent la guerre et la chasse, où, contrairement au tir de compétition, les conditions d'utilisation sont proches de celles que connaissaient les Grecs de l'Antiquité.

111 On imagine mal, par ex., que, dans le champ de leur mètis spécifique, les cinq tokosowoko mycéniens, c'est-à-dire tołofoproí, facteurs d'arcs, mentionnés par une tablette de Pylos (An 20712), aient pu ignorer les performances du frêne.

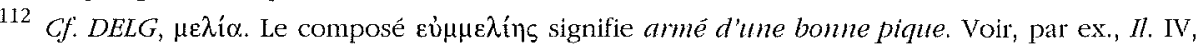
47 et VI, 449 , à propos de Priam.

113 Respectivement, XVI, 114; XX, 272; XXI, 293 et XVI, 140-144 (= XIX, 388-391). Quand Achille prête ses armes à Patrocle, lá seule que celui-ci ne prend pas est la pique en frêne du Pélion $(\pi \eta \lambda$ ıó $\delta \alpha \mu \varepsilon \lambda i ́ n v, X V I, 143)$ : le centaure Chiron l'a fabriquée et offerte à Pélée; Achille en a hérité et lui seul a la force de la brandir. 
l'épieu du chasseur ${ }^{114}$. Bois de lance et aussi, comme je le propose, d'arc et de flèche, le frêne est, en Grèce ancienne, chargé d'une symbolique marquée par la guerre, la violence : c'est du frêne que le mythe des races raconté par Hésiode fait descendre le troisième génos créé par Zeus, la race de bronze ${ }^{115}$.

Devenu arc, flèche, lance, le frêne découvre son humeur combattante. Armé de sa pointe de bronze, le bois de frêne est un bois qui pique, qui transperce, qui tue, à la façon précisément des animaux-archers qu'il met en fuite à Claros.

L'écologie du frêne vient éclairer et compléter cette vision : elle dresse le portrait d'un arbre réputé faire le vide autour de lui pour se développer. Etudiant ce qu'il appelle le « comportement social » du frêne, G. Motel décrit un grand consommateur d'eau qui peut entraîner la mort de la végétation la plus proche : "Adulte, le frêne supporte difficilement la concurrence des autres végétaux et il les élimine rapidement sous son couvert ${ }^{116}$. Mais le frêne a pu être incriminé pour d'autres raisons : «Si le sol est presque nu sous les frênes, certains affirment que ceci est dô aux pluies qui entraînent des produits nocifs émis par les feuilles, produits qui empoisonneraient les autres plantes ${ }^{117}$. Peut-être y a-t-il un rapport entre cette suspicion d'empoisonnement - qui semble infondée ${ }^{118}$ - , et l'attirance bien réelle que le frêne exerce sur deux insectes «toxiques". Redouté pour ses dangereuses piqûres, le frelon (Vespa crabro) apprécie le frêne auquel il peut causer d'importants dégâts ${ }^{119}$. Quant à la cantharide, Pline souligne sa prédilection pour le frêne ${ }^{120}$ : ce petit coléoptère (Lytta vesicatoria $\mathrm{L}$.) en dévore les feuilles et produit une substance malodorante et irritante, la cantharidine, qui peut être très toxique si elle est inhalée. De même, réduite en poudre, la cantharide a été utilisée pour confectionner des poisons violents et des remèdes ${ }^{121}$. De tels « hôtes » ont pu renforcer l'idée que le frêne était en quelque sorte venimeux. Le frêne producteur ou porteur de poison ? Voilà qui vient compléter la représentation que les Grecs ont pu avoir de cet arbre: un arbre aux flèches enduites de poison, tout aussi «toxiques » que celles décochées par les bêtes à venin qu'il met en fuite à Claros.

114 Voir V.D. HANSON, Le modèle occidental de la guerre. La bataille d'infanterie dans la Grèce classique, Paris, Les Belles Lettres, 1990 [New York, 1989], p. 120, 123; J.K. Anderson, "Equipement hoplitique et armes offensives" [Londıes, 1991], in P. Brulé et J. Oulhen (éds), La glterre en Grèce à l'époque classique, Rennes, PUR, 1999 (Histolre), p. 119-120 et M.M. MARKLE, "La sarisse macédonienne, la lance et l'équipement connexe" [AJA 81, 1977], ibidem, p. 151.

115 Les Travaux el les Jours, 143-155. Elle est fille des frênes, terrible et puissante (غ́к $\mu \varepsilon \lambda_{1} \hat{\alpha} v$, $\delta \varepsilon$ vóv $\tau \varepsilon \kappa \alpha i ̀ ~ o ̈ \theta p ı o v, 145)$, ne pense qu'à la guerre et finit par s'entretuer.

116 O.c. (n. 74), p. 11. Voir aussi p. 13 et 49. Duflot, o.c. (n. 74), p. 27, décrit la concurrence impitoyable que les jeunes frênes subissent de la part des grands frênes assoiffés (p. 22-23). Bertrand, o.c. (n. 74), p. 37, relève aussi cette réputation « d'empêcheur de pousser en rond » qui poursuit le frêne trop gourmand en eau.

117 Motel, o.c. (n. 74), p. 39. Voir aussi Durlot, o.c. (n. 74), p. 162.

118 Voir J. Bruneton, Plantes toxiques. Végétaux dangereux pour l'Homme et les animaux, Lonclres, Technique \& Documentation, 1999 [1996] : pas de mention du frêne dans cet ouvrage.

119 Voir Duflot, o.c. (n. 74), p. 45; Bertrand, o.c. (n. 74), p. 24 et Motel, o.c. (n. 74), p. 43-44.

${ }^{120}$ HN XXIX, 94. Voir à ce propos Motel, o.c. (n. 74), p. 40-41 et Bertrand, o.c. (n. 74), p. 26.

121 Bertrand, o.c. (n. 74), p. 28, prend comme exemple le poison des Médicis. Les médecins et naturalistes de l'Antiquité connaissent bien le pharmakon ou le venenum de la cantharide. Cf. Pline (HN XI, 118 et XXIX, 93-94, 96), Dioscoride (De materia medica II, 61, 1-2) et Galien (Kühn XII De semplicium medicamentonum temperamentis et facultalibus IX, 2, p. 171 sq.). 
Mais le frêne n'est pas seulement un archer aux flèches meurtrières et venimeuses. Il est aussi protecteur et sauveur, ce dont témoignent, de façon très explicite, les quatre vers de Nicandre : ce sont bien les frênes, investis de cette tâche par Apollon, qui mettent le sanctuaire de Claros à l'abri des piqûres et des venins. Pline se fait l'écho d'une longue tradition d'incompatibilité entre le frêne et le serpent, un des trois pensionnaires du bestiaire clarien ${ }^{122}$. Cet antagonisme, qui repose peut-être sur une certaine réalité ${ }^{123}$, se retrouve dans de nombreuses traditions. Il est très probablement à l'origine de l'emploi du frêne comme antidote en cas de morsure de vipère $^{124} . \mathrm{B}$. Bertrand décrit une véritable «médecine du frêne », réputée favoriser la longévité et encore très active jusqu'à la Seconde Guerre Mondiale : l'action diurétique et dépurative, des infusions de feuilles notamment, explique qu'elles étaient utilisées pour éliminer les poisons ${ }^{125}$.

Les qualités médicinales, et plus particulièrement antivenimeuses du frêne, s'accordent parfaitement avec ce qu'Élien dit d'Apollon à Claros : le dieu que les bêtes à flèches et à venin redoutent, y est honoré comme celui qui sait sauver, comme le père d'Asclépios, sauveur et combattant des maladies. Ainsi le frêne qui pique, transperce et peut-être empoisonne, qui fait place nette autour de lui, est aussi un frêne-antidote, qui écarte le mal causé par les piqûres venimeuses et les poisons : Epikourios, qui aide, qui porte secours, qui protège, comme l'est Apollon ${ }^{126}$, il mérite tout autant que lui les épiclèses «thérapeutiques » de Pbarmakios, qui porte remède ${ }^{127}$, de Iatros, Médecin et d'Akésios, Guérisseur ${ }^{128}$.

Cette ambivalence fondamentale du frêne, à la fois meurtrier et médecin, est aussi celle de l'arc, de la flèche et du dieu qui les porte. On rejoint ici la problématique exprimée par Héraclite dans un des aphorismes antithétiques qu'il affectionne ${ }^{129}$ :

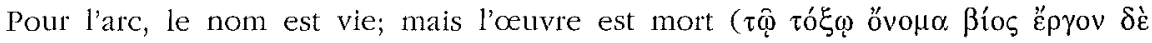

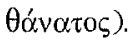

${ }^{122}$ Cf. HN XVI, 64 : bu ou pris comme topique, le jus de feuilles de frêne guérit les morsures de serpent. Les serpents fuient l'ombre et le feuillage de l'arbre. Voir Diosc., De mat. med. I, 80.

123 Cf. J. ANDré, n. 3, p. 121 à XVI, 64 in Pline, Histolre Naturelle, Paris, Les Belles Lettres, 1962.

124 Cette utilisation thérapeutique a longtemps perduré dans la pharmacopée populaire des campagnes européennes. Voir ANDRé, o.c. (n. 123), n.2, p. 120-121 à HN XVI, 64.

${ }^{125}$ La médecine d'aujourd'hui reconnaît l'efficacité de nombreux traitements à base de frêne. Voir Bertrand, o.c. (n. 74), p. 48-52 ainsi que L. Bézanger-Beauquesne, M. Pinkas, M. Torck et Fr. Trotin, Plantes médicinales des régions tempérées, Paris, Maloine, 1992 [1980], p. 231-232.

${ }^{126} C f . D E L G$, éríkovpos. Sur l'origine, la signification et l'évolution de cette épithète qui qualifie Apollon comme bienfaiteur des hommes, il faut voir M. Josr, Sanchatres et culles d'Arcadie, Paris, EFA, 1985 (Études Péloponésiennes, 9), p. 222-223, 485-489, 555. Il est vraisemblable que l'Apollon Secourable de Bassai en Arcadie, fut d'abord un dieu très militaire, avant d'élargir son aide à tous ceux qui en avaient besoin, au point que Pausanias (VIII, 41, 8-9) a pu en faire l'équivalent de l'Alexikakos si salutaire en temps de 'pestes'.

127 C'est une des nombreuses épiclèses d'Apollon à Rhodes, à Camiros précisément, où elle est attestée par une inscription (TC $\left.{ }^{*} 90,1,30\right)$ : le prêtre d'Apollon Pbarmakios est mentionné dans une liste du $\mathrm{Il}^{\mathrm{e}} \mathrm{s}$. av. J.-C. ( ca 164). Le dieu est alors celui qui peut remédier au mal ou le remède (phamakon) lui-même. Cf. D. Moreli, I culti in Rodi, Pise, 1959 (SCO, 8), p. 28, 102-103, 110.

128 Sur cet aspect d'Apollon, cf. Derienne, o.c. (n. 72), p. 201, 208, 229 et n. 344, p. 330.

129 Traduction de M. Conche dans Héraclite, Fragments, Paris, PUF, 1991 [1986], (Épimétbée), p. 423, fr. 124 Conche $=48$ Diels-Kıanz, donné par l'Etymologicum Magnum, s.v. ßı́s. Je renvoie, p. 424 , à la pertinente analyse de ce fragment. 
En jouant sur l'homonymie presque parfaite entre $\beta$ rós, l'arc ${ }^{130}$ et $\beta$ '́os, la vie ${ }^{131}$, la formule énonce un paradoxe qui n'est qu'apparent. Dans les mains de l'archer humain comme de l'archer divin, l'arc et les flèches sont certes des instruments de mort, mais ils sont aussi, par là même, des armes de vie : l'archer vit de la mort qu'il décoche. C'est vrai du chasseur qui tue pour vivre, ce que le Philoctète de Sophocle montre de façon exemplaire, en jouant lui aussi sur l'homonymie $\beta$ iós $/ \beta$ íos $^{132}$. C'est vrai aussi du guerrier : quand il inflige la mort avec son arc, il protège sa vie et celle de ses compagnons. Faire œuvre de vie en décochant ses flèches est un des arguments du défenseur de l'archer sur le champ de bataille, dans le débat contradictoire de l'Héraclès d'Euripide (v. 195-197) ${ }^{133}$. C'est vrai enfin pour Apollon, comme en

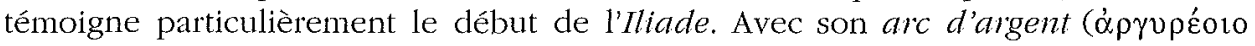
$\beta$ iồ, I, 49) dont le nom évoque la « vie », le dieu en colère fait ceuvre de mort : il décoche sur les Grecs ses flèches «pestilentielles » qui portent le terrible loimos (v. 61) ${ }^{134}$. Or, quand Apollon décoche ses flèches pathogènes et mortifères, il « vit d'autant plus intensément sa vie divine ${ }^{135}$ : en faisant œuvre de mort pour les hommes, l'arc fait donc cuvre de vie pour le dieu. L'Archer qui déclenche les fléaux à coups de flèches est aussi celui qui les écarte au moyen de ses propres flèches et le médecin qui sait les guérir : archer et médecin, ainsi se présente Apollon, à Didymes,

${ }^{130}$ Cf. DELG, $\beta$ tóç : « le mot est ancien mais près de disparaître. 》 Chantraine signale qu’à l'exception de ce fragment, il est strictement homérique et cinq fois moins employé chez Homère que le mot usuel tóķov.

131 Cf. $D E L G$, Bíos : ainsi accentué, le mot signifie par dérivation, 'moyens de vivie', 'ressources'.

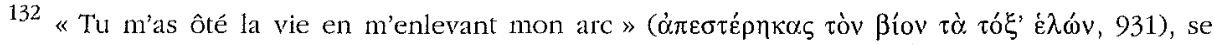
plaint Philoctète à Néoptolème (trad. P. Mazon). Plus loin, le terme $\tau \rho \circ \varphi n ́$ (v. 1126) désigne l'arc comme 'ce qui procure de la nourriture', 'ce qui fait vivre' et le texte met souvent en évidence que l'arc est le seul moyen de subsistance de Philoctète. Voir P. VIDAL-NAQUET, "Le Philoctète de Sophocle et l'éphébie", in Mythe et tragédie en Grèce ancienne I., Paris, 1989 [1972], p. 170-171.

133 « Au contraire, celui dont le bras est habile à tirer de l'arc possède cet avantage unique de

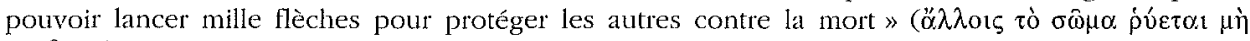

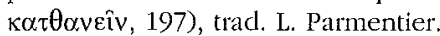

${ }^{134}$ Les historiens de la médecine ne reconnaissent pas la 'peste' proprement dite dans le loimos, et préfèrent parler de 'pestilences'. Cf. М. Grmeк, Les maladies à l'aube de la civilisation occidentale. Rechercbes sur la réalité patbologique dans le monde grec bistorique, arcbaïque et classique, Paris, 1994 [1983], p. 33 et Jouanna, o.c. (n. 36), p. 290. La pensée mythique et prérationnelle a longtemps attribué aux flèches d'Apollon la cause de ces maladies épidémiques qui frappent subitement et massivement. Voir Detrenne, o.c. (n. 72), p. 124 -125, 227-229 et n. 176, p. 321 notamment : de façon générale, les maladies et les autres 'fléaux' divins - famine, stérilité du sol, guerre civile, dérèglement de la reproduction... - sont le signe, qui a pu être envoyé par Apollon ou Artémis, d'une souillure restée sans purification. Par ses oracles, Apollon peut révéler le sens de ce signe et comment y porter remède. Sur cette conception religieuse qui voit dans le fléau pestilentiel la punition divine infligée par un Apollon offensé, voir aussi M. Grmek et D. Gourevitch, Les maladies dans l'art antique, Paris, Fayard, 1998, (Penser la médecine), p. 75, 93, 95-96. Elle est combattue par la médecine hippocratique qui accuse les 'miasmes', ces exhalaisons délétères véhiculées par l'air. Concernant cette divergence étiologique, on peut se reporter à l'analyse de Jounnna, o.c. (n. 36), p. 292-293, ainsi qu'à Grmek et Gourevi'ch, ibidem, p. 93. Les pathologies qui affligeaient souvent les armées en campagne se prêtaient tout particulièrement, me semble-t-il, au rapprochement avec l'image de la flèche en vol dans un champ de bataille: ce mal qui neuvre, silencieux et invisible, qui frappe à l'improviste, qui tue en plusieurs endroits à la fois et en même temps, un grand nombre de personnes, ressemble à la mort, elle aussi invisible et silencieuse, que les flèches semblent semer au hasard dans la masse des guerriers.

135 CONCHE, o.c. (n. 129), p. 424. 
dans un de ses plus anciens oracles daté de 550-525 avant J.-C., découvert dans la colonie milésienne d'Olbia, sur la rive nord du Pont-Euxin. Gravé sur une tablette en os, le texte oraculaire évoque Apollon, Archer, amical par les dons qu'il fait

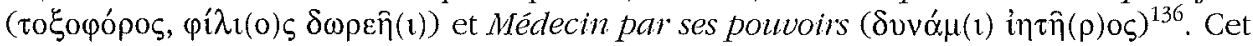
archer qui peut être meuttrier et salutaire, est aussi le Devin (Mantis) vers lequel les hommes se tournent quand frappent la mort et la maladie. Consulté par la cité phrygienne de Hiérapolis qu'accable l'épidémie de 166 sous le règne de Marc Aurèle, l'oracle de Claros répond:

Consacrez devant toutes les portes, dans un téménos, une sainte statue de Phoibos

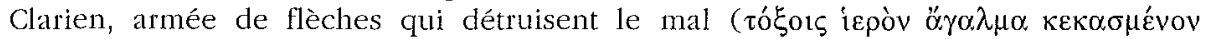
$\hat{\omega} \lambda \varepsilon \sigma t v o v \sigma o 1 \varsigma$ [19]), pour que de ses traits il chasse au loin le loimos insatiable (oiov

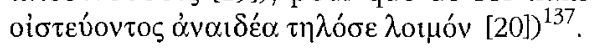

En médecin, le devin de Claros délivre un oracle-ordonnance qui prescrit contre le loimos un remède d'archer ${ }^{138}$. L'Archer, le Médecin et le Devin se confondent : c'est un véritable médicament, un pharmakon que les consultants semblent attendre du dieu, et contre la maladie sagittaire, l'Archer recommande un pharmakon sagittaire. Or, la flèche et l'arc qui la décoche, partagent avec le pharmakon la même ambiguité : comme lui, aussi bien remède que poison - en fonction du dosage de la drogue ou de l'honnêteté du médecin - ${ }^{139}$, l'arc et la flèche peuvent être des instruments de vie ou de mort et le parallèle est d'autant plus frappant que la flèche peut être elle-même enduite de pharmakon, empoisonnée. Ce sont alors l'archer et le médecin eux-mêmes qui, sous le patronage d'Apollon, apparaissent comme de proches parents, pouvant faire ouvre de vie comme ouvre de mort au moyen de leur flèche-pharmakon. Dans les mains de l'archer, l'arc et lá flèche changent donc de signe et livrent leur double nature qui réunit deux valeurs radicalement contraires en apparence: la mort et la vie. Ce sont ces mêmes valeurs que partagent ceux qui portent ces instruments : le chasseur, le guerrier, Apollon et les deux protagonistes clariens que nous avons étudiés, le scorpion et le frêne. Le scorpion incarne le côté noir de la flèche et fait essentiellement oeuvre de mort, même s'il est réputé pouvoir

136 Je renvoie aux analyses et aux suggestions de W. Burkert, "Olbia and Apollo of Didyma: A New Oracle Text", in J. Solomon (éd.), Apollo. Origins and Influences, Tucson, The University of Arizona Press, 1994, p. 49-60, qui étudie l'intégralité de cet énigmatique oracle digne de Loxias. Pour l'aspect qui nous intéresse ici, voir les pages 52-55.

137 voir G. Pugliese Carratelli, "XPhrmol di Apollo Kareios e Apollo Klarios a Hierapolis in Frigia", ASAA 41/42 (1963/1964), p.360-365. J'ai traduit les trois hexamètres (1. 18-20 de l'inscription II b) sur le texte grec (p. 360) en m'appuyant sur la traduction italienne de l'auteur (p. 362). Voir aussi DetTENNe, o.c. (n. 72), p. 124

${ }^{138}$ La consultation oraculaire prend ici des allures de consultation médicale et on pense à l'Apollon Iatromantis d'Eschyle (Euménides, 63), le dieu qui guérit par ses oracles. A propos de Calchas, le devin d'Apollon qui révèle aux Grecs la cause du loimos et comment y porter remède (II. I, 62-100), Jouanna fait bien ressortir la ressemblance entre le pronostic médical et la prophétie, o.c. (n. 36), p. 145-147.

139 Sur l'ambiguité du concept de pharmakon, on peut voir D. Gourevitch, Le triangle bippocratique dans le monde gréco-romain. Le malade, sa maladie et son médecin, Paris, EFR, 1984 (BEFAR, 251), p. 367-371, et JouAnNa, o.c. (n. 36), p. 185-186. Il est remarquable que cette ambiguïté recoupe celle du médecin : à côté du bon médecin paré de toutes les vertus, Gourevitch dresse le portrait de l'anti-hippocrate', le mauvais médecin toujours soupçonné d'être un empoisonneur potentiel par manque de scrupules ou de compétence (p. 348-374). 
guérir le mal qu'il cause. Le frêne fait fondamentalement œuvre de vie, en repoussant le mal qui menace, mais il le fait en brandissant dans ses frondaisons des instruments de mort. Sous le signe ambivalent de la flèche apollinienne qui agit comme un pharmakon, tous deux incarnent les valeurs négatives et positives des instruments de l'archer.

Au terme de ce second volet, très « mélien », de notre étude, on saisit mieux les raisons pour lesquelles, à Claros, les Grecs ont choisi un bois de frênes pour abriter le sanctuaire du dieu de la mantique, du dieu archer, meurtrier et médecin ${ }^{140}$. Le frêne évoque la séance oraculaire avec absorption d'eau au plus secret du temple : comme le prophète qui descend dans l'adyton, le frêne plonge ses racines dans le « site en adyton " qu'est le " profond vallon " clarien et boit l'eau sacrée d'Apollon. Le frêne évoque aussi les instruments de l'Archer : ses branches sont chargées de pointes de flèches et son bois, apprécié pour la lance, a très bien pu servir pour fabriquer arcs et flèches en pays grec. Le frêne évoque encore les pouvoirs de l'Archer': comme l'Archer, le frêne tient à l'écart des agresseurs porteurs de mort ou de maladie; comme l'Archer, il protège et guérit des maux sagittaires qui lui ressemblent. Un arbre en quelque sorte inspiré par Apollon, un arbre archer, antidote et médecin, tel apparaît le frêne clarien, digne de figurer en bonne place dans l'arboretum divin, aux côtés du palmier ou du laurier. La frênaie clarienne n'est donc pas un simple décor naturel autour du sanctuaire, mais bien plutôt un lieu habité par le pouvoir d'Apollon et dans lequel ce pouvoir s'exerce ${ }^{141}$. C'est à un alsos défensif, hérissé de frênes-archers, puisant leurs forces dans l'eau sacrée, que se heurtent d'autres archers indésirables, les bêtes à flèches et à venin qui veulent pénétrer dans le sanctuaire.

Ainsi, bien au-delà de la petite tradition locale d'apparence anodine, les quatre vers de Nicandre cités par Élien, s'insèrent dans un complexe apollinien foisonnant mais très cohérent, articulé autour de l'arc et des flèches, et de la charge symbolique dont ils sont porteurs dans les mains du dieu archer.

\section{CRESCAM}

Philippe MonBrun

Université de Rennes II

6, avenue Gaston Berger

F - 35043 Rennes Cedex.

140 «Les Grecs ont choisi »: cette formulation, comme d'autres équivalentes utilisées dans cet article, exclut que le frêne puisse se trouver dans le sanctuaire sans avoir de rapport particulier avec Apollon. Elle laisse entendre au contraire que l'arbre et le dieu vont ensemble, même si les deux distiques de Nicandre sont peut-être seuls à en témoigner de façon explicite. Qu'un bois de frênes antérieur au choix du lieu de culte ait «appelé » la présence d'Apollon ou bien que le dieu ait " appelé » ce bois, ce sont bien les Grecs qui, à Claros, « ont choisi » de réunir Apollon et le frêne.

141 Aussi éprouvai-je quelque difficulté pour appliquer, au cas particulier de Claros, le jugement suivant de JACOB, l.c. (n. 16), p. 44 : « Tout nous suggère que l'ő $\lambda \sigma o \varsigma$ est davantage un cadre paysager qu'une manifestation de la présence du divin, un décor végétal entourant le sanctuaire proprement dit plus qu'un lieu sacralisé dans son essence même. » 


\section{Illustrations}

Doc. 1a et b : Araignée, serpent et scorpion en relief sur une amulette d'Hagia Triada. D'après M. Mastorakis, M. Van Effenterre, Les Minoens. L'âge d'or de la Crète, Paris, 1991, p. 162.

Doc. 2 : Sceau au scorpion de Mallia. D'après Poursat, o.c. (n. 14), fig.11, p. 23.

Doc. 3a : L'arc dit « droit 》 (d'après sa position au repos). D'après E. MCEwEN, R. MiLler et Chr. Bergman, "La conception et la fabrication des arcs anciens", Pour la Science 166 (août 1991) [éd. française de Scientific American], p. 76.

Doc. 3b : L'arc « réflexe » ou palintone. D'après G. Rausing, The bow. Some notes on its origin and development, Bonn/Lund, 1967 (Acta Arcbeologica Lundensia 6), album, fig. 2.

Doc. 4a et b: Le scorpion, une catapulte à flèches. D'après J. WARrY, Histoire des guerres de l'Antiquité, Bruxelles, Elsevier Séquoia, 1981 [Londres, 1980], p. 78.

Doc. 5 : La « flèche du Pauthe ». D'après Roth, o.c. (n. 103), fig. 83, p. 86.

Doc. 6 : La feuille composée du frêne oxyphyle. D'après Motel, o.c. (n. 74), p. 24.

Doc. 7 : Couler au moule des pointes de flèches : une lecture «verte ». Bronze Récent, Musée de Chypre, D'après Hult, l.c. (n. 96), fig. 183, p. 93 ( $\left.\mathrm{n}^{\circ} \mathrm{N} 1055\right)$.

Doc. 8a : Pointe de Hala Sultan Tekké. Bonze récent. D'après Hult, l.c. (n. 96), fig. 149, p. 90 (n N 1114).

Doc. $8 \mathbf{b}$ et $\mathbf{c}$ : Type (VIIa) de pointe égéenne de l'âge du Bronze. D’après BuchHolz, l.c. (n. 100), fig. 7, p. 11 et $13 a-c$, e et i, p. 22. 